\title{
Desarrollo metodológico para la construcción de escenarios urbano-energéticos de largo plazo
}

\section{Pedro Chévez}

Becario posdoctoral del CONICET. Arquitecto por la UNLP, especialista en Energías Renovables por la UNSa y doctor en Ciencias (Área Energías Renovables) por la UNSa. Integrante del Instituto de Investigaciones y Políticas del Ambiente Construido (CONICET-UNLP). chevezpedro@ gmail.com.

\section{Irene Martini}

Investigadora adjunta del CONICET. Arquitecta por la UNLP, magíster en Políticas Ambientales y Territoriales por la UBA y doctora en Ciencias (Área Energías Renovables) por la UNSa. Subdirectora del Instituto de Investigaciones y Políticas del Ambiente Construido (CONICET-UNLP). irenemartini@conicet.gov.ar.

\section{Carlos Discoli}

Investigador principal del CONICET. Ingeniero Mecánico por la UTN, magíster en Ambiente y Patología Ambiental por la UNLP (en convenio con la Escuela de los Altos Estudios de Siena) y doctor en Ciencias (Área Energías Renovables) por la UNSa. Integrante del Instituto de Investigaciones y Políticas del Ambiente Construido (CONICET-UNLP).

discoli@rocketmail.com.

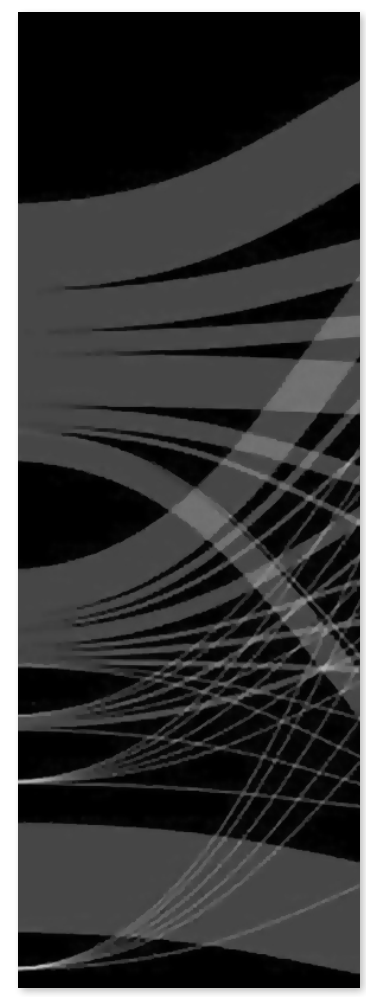




\section{Desarrollo metodológico para la construcción de escenarios urbano-energéticos de largo plazo}

\section{Desenvolvimento metodológico para a construção de cenários de energia urbana a longo prazo}

\section{Resumen}

Este trabajo presenta el desarrollo metodológico para la construcción de escenarios urbano-energéticos de largo plazo (2014-2040) a partir de la implementación de estrategias de eficiencia energética y energías renovables. Dichos escenarios consideran tanto la oferta como la demanda de energía, focalizando sobre el sector residencial y en las diferencias intra-urbanas que este presenta. Se utiliza como caso de estudio la ciudad de La Plata (Argentina). Se exponen los aspectos teórico-conceptuales, los métodos, técnicas, procedimientos, variables e hipótesis necesarias para su desarrollo a los efectos de que la presente metodología pueda ser replicada en otros casos de estudio.

\section{Palabras clave}

Escenarios urbano-energéticos; sector residencial; urbano; demanda; oferta.

\begin{abstract}
This work shows the methodological development for the construction of long-term urban-energetic scenarios (2014-2040) from the implementation of energy efficient and renewables energies strategies. These scenarios consider both the supply and the energy's demand, making focus over the residential sector and over the intra-urban differences that it shows. La Plata (Argentina) city is used as study case. Theorical-conceptual aspects, methods, techniques, procedures, variables and hypothesis necessaries to its development, are exposed here with the purpose of replicating this methodology in other study cases.
\end{abstract}

\section{Keywords}

Urban-energy scenarios; residential sector; urban; demand; supply.

\section{Resumo}

Este trabalho apresenta o desenvolvimento metodológico para a construção de cenários de energia urbana de longo prazo (2014-2040) a partir da implementação de estratégias de eficiência energética e energias renováveis. Esses cenários consideram tanto a oferta quanto a demanda de energia, com foco no setor residencial e nas diferenças intra-urbanas que este apresenta. A cidade de La Plata (Argentina) é usada como estudo de caso. Os aspectos teórico-conceptuais, os métodos, as técnicas, os procedimentos, as variáveis e as hipóteses necessárias para seu desenvolvimento são expostas aqui a os efeitos de que esta metodologia possa ser replicada em outros casos de estudo.

\section{Palavras chave}

Cenários de energia urbana; setor residencial; urbano: demanda; oferta. 


\section{Introducción}

\subsection{La problemática energética y la necesidad de una respuesta urbana}

La energía puede definirse como un bien estratégico que satisface necesidades sociales y tiene incidencia directa sobre el sector industrial, por lo cual se trata de un recurso fundamental para el desarrollo socioeconómico de cualquier país. Los modos de producción y consumo de energía repercuten de manera directa sobre la economía, las ciudades, sus habitantes, el ambiente y la política; por tales motivos, su tratamiento ocupa un rol protagónico en el diseño e implementación de políticas públicas (Guzowski, 2015). La energía en general y los combustibles fósiles en particular son pilares fundamentales para movilizar el actual sistema capitalista y, por ello, su desabastecimiento supone una amenaza a los procesos de acumulación. Por tal motivo, su provisión y sus formas de consumo ocupan posiciones de importancia en la agenda política global.

Sin embargo, tanto a nivel mundial como en Argentina, el consumo y abastecimiento de combustibles fósiles presentan una situación compleja. Este hecho genera inconvenientes asociados a la volatilidad de sus precios y su consecuente impacto sobre la economía. A su vez, se presentan tensiones geopolíticas permanentes por el control de los recursos y, al no haber certezas sobre los niveles de reservas futuras, existe un consenso generalizado en señalar la necesidad de reducir los niveles de consumo, situación que se ha acentuado con los efectos del cambio climático consecuente de las emisiones de gases de efecto invernadero. 
En función de lo expuesto, se presenta un escenario donde es ineludible replantear dicha dependencia, debiendo, en consecuencia, establecer acciones orientadas al mejoramiento de la eficiencia energética en los usos finales y la sustitución de fuentes convencionales por renovables. Esto permitirá a futuro modificar la matriz energética y dar respuestas al mencionado problema. Por su parte, en el ámbito urbano existe una tendencia global incipiente donde se observa que las ciudades han empezado a asumir la responsabilidad en dicha problemática, impulsando programas de inserción de energías renovables a escala urbana o promoviendo mejoras en la eficiencia energética tanto de los edificios nuevos como de los existentes, entre otras acciones (CORTEKAR ET ÁL., 2016; WEC, 2016).

En el caso de la Argentina, existen diversas problemáticas energéticas, consecuencia de las sucesivas transformaciones políticas ocurridas durante las últimas décadas, las cuales han definido el actual sistema eléctrico e hidrocarburífero. En el caso del sector eléctrico, en el área de la distribución, existen cortes de suministro masivos principalmente en verano (Decreto 134, 2015); mientras que en el sector de generación, no se alcanzan a cubrir los picos de demanda y la matriz es altamente dependiente de los combustibles fósiles (RugGERI, 2017). Simultáneamente, en el sector hidrocarburífero, se presenta un contexto con un declive sostenido en la producción de petróleo y gas (BARRERA ET ÁL., 2012), lo que obliga al Estado a destinar importantes sumas de dinero en importaciones de combustibles. En este sentido, en la Argentina el sector residencial posee un gran impacto sobre la demanda total, dado que en 2014 requirió un 25,98 $\%$ del total (15921 kTEP-1,18 TEP/vivienda-). Dicha participación se complementa con el sector industrial (24,98 \%), transporte (27,94\%), comercial y público (7,76 \%), agropecuario (6,98 \%) y no energético (6,76 \%) (MINEM, 2016). Por su parte, es necesario destacar que un 90,28\% de las viviendas argentinas están localizadas en áreas urbanas (INDEC, 2016). Es por ello que si se focalizaran los esfuerzos en reducir las demandas en las ciudades, a partir de medidas de eficiencia energética (EE) y de la incorporación de energías renovables (ER) en el sector residencial, sería viable alcanzar reducciones significativas en la demanda total y minimizar el impacto de las problemáticas asociadas al abastecimiento de energía.

En este contexto, los estudios urbano-energéticos que contemplen la oferta y la demanda, sobre todo los de mediano y largo plazo como los "escenarios", se posicionan como instrumentos de vital importancia para la evaluación y formulación de políticas energéticas, dado que posibilitan gestionar desde una óptica integrada las interacciones entre los principales vectores energéticos y los sectores de consumo. 
Teniendo en cuenta la necesidad de implementar este tipo de estudios en el ámbito urbano, cabe señalar que, en general, los trabajos de escenarios energéticos no desagregan las diferencias que existen dentro de la estructura urbana y suelen abordar el área de estudio en su totalidad como un elemento homogéneo. Si bien se encuentran trabajos que analizan las potencialidades de sectores diferentes de la ciudad, hacen referencia a un único tipo de acciones, tales como el reciclado edilicio masivo (RodRIGUEZ ET ÁL., 2016) o el uso de energía solar (EICKER ET ÁL., 2014; BRITO ET ÁL., 2017). Además, dichos estudios no logran abordar la dimensión temporal. Por su parte, existen experiencias en las que se estudia la incorporación de estrategias energéticas en ámbitos urbanos con un enfoque de largo plazo, utilizando escenarios, pero que por ejemplo adoptan como unidad de análisis las tipologías de vivienda para ensayar medidas de mejoramiento energético, lo cual impide la interacción con variables territoriales (DroullLes ET ÁL., 2017; GHiassi \& MAHDAvi, 2017). También existen estudios que abordan los usos energéticos de los distintos sectores urbanos, pero que únicamente analizan su demanda futura de forma global, sin posibilidades de ensayar medidas energéticas de forma precisa (HowARD ET ÁL., 2012). Otros trabajos analizan el impacto de medidas puntuales como la integración de un sistema de calefacción distrital o medidas de mejoramiento de la envolvente edilicia en sectores urbanos reducidos, lo cual impide identificar las diferencias intra-urbanas del caso de estudio (GirARdin ET ÁL., 2010; ABDURAFiKov ET ÁL., 2017). En tanto, se encuentran trabajos de escala urbana que contribuyen directamente a los planificadores y tomadores de decisiones, en los que se evalúan energéticamente las futuras expansiones urbanas, cuyo objetivo no es el ensayo de medidas de mejoramiento (SveInbjörnsson ET ÁL., 2017; Mörtberg ET ÁL., 2016).

En consecuencia, se observa que es poco frecuente encontrar estudios que incluyan simultáneamente múltiples aspectos que relacionen e integren la reducción de la demanda de energía a través de la aplicación de medidas de eficiencia energética de la envolvente edilicia, el recambio de equipamiento domiciliario, la inserción de energías renovables y que, al mismo tiempo, desagreguen la información por áreas urbanas de comportamiento homogéneo. Por esta razón, los trabajos de investigación de VIEgAS (2010) o AMADO ET ÁL. (2016) son de importancia, dado que proporcionan una metodología de detección y clasificación masiva de tipologías edilicias y de los materiales constructivos para el mejoramiento de la envolvente de los edificios y el potencial solar en diferentes sectores urbanos; sin embargo, su análisis no presenta un alcance temporal de largo plazo. 
La revisión bibliográfica permite advertir la existencia de diferentes enfoques para la elaboración de diagnósticos urbano-energéticos. En general, los trabajos se focalizan en un número limitado de medidas de mejoramiento, abordan la ciudad en su conjunto o se restringen a sectores urbanos reducidos, y no siempre se toma en consideración el enfoque de largo plazo. En este sentido, no se logran abordajes integrales que consideren simultáneamente las diferentes estrategias posibles de implementar en la ciudad, las diferencias intra-urbanas que permitan identificar la criticidad de las distintas áreas que la conforman y que, a su vez, tengan en cuenta las dinámicas de crecimiento urbano y las trayectorias energéticas de largo plazo.

\subsection{Objetivos y alcances del trabajo}

En función de la problemática planteada y de la revisión bibliográfica, el presente trabajo desarrolla una metodología versátil orientada a la construcción integrada de escenarios urbano-energéticos. Dicha metodología considera tanto la oferta como la demanda de energía, tiene en cuenta el impacto individual y simultáneo de las distintas medidas de mejoramiento energético posibles de aplicar en función de las necesidades y posibilidades de los diferentes sectores de la ciudad y, además, permite cuantificar los ahorros posibles de alcanzar en el largo plazo. Así, se contribuye al desarrollo de una planificación energética integral —a nivel urbano—y detallada — para cada una de las áreas homogéneas analizadas-.

En consecuencia, este trabajo tiene como objetivo exponer detalladamente los aspectos metodológicos, a los efectos de posibilitar la evaluación y validación de los distintos métodos, técnicas y datos utilizados. Se presenta una instrumentación "abierta” y susceptible de ser mejorada, ampliada y ajustada con la inclusión de nuevos datos. Esto permitirá contrastar y/o dar mayor precisión a los resultados, así como facilitar la adopción de la metodología a otros casos de estudio. Cabe destacar que la metodología aquí presentada fue desarrollada y ensayada en el marco de la tesis doctoral titulada Construcción de escenarios urbano-energéticos a partir de la implementación de estrategias de eficiencia energética y energías renovables en el sector residencial (CHÉvez, 2017). 


\section{Método de abordaje general: anclaje territorial,} etapas y aproximación al universo de análisis

En esta sección se describen sintéticamente el caso de estudio utilizado como ejemplo de aplicación, las etapas necesarias para desarrollar la construcción de escenarios urbanoenergéticos y el método de abordaje del universo de análisis.

\subsection{Anclaje territorial}

Si bien este trabajo presenta un desarrollo metodológico teórico, utiliza un ejemplo de aplicación concreto. En consecuencia, el anclaje territorial del universo de análisis se realiza en la ciudad de La Plata, capital de la provincia de Buenos Aires (Argentina). La ciudad contaba en 2014 con una población estimada de 631.831 habitantes y, si bien cuenta con vínculos estrechos con la Región Metropolitana de Buenos Aires, posee una dinámica de funcionamiento propia, definiéndose como una "ciudad intermedia”. Este tipo de ciudades responden a cierta autonomía, pero a su vez son dependientes de las grandes metrópolis, realizan funciones de intermediación entre núcleos más pequeños y grandes áreas metropolitanas, se caracterizan por presentar cierta complejidad funcional, por tener un grado importante de centralidad y poseer significativos elementos de simbología histórica, infraestructura y servicios (RodríguEz, 2016). En este sentido, su análisis presenta una gran potencialidad de replicabilidad en universos de similares características, ya que este tipo de ciudades son las de mayor predominancia en nuestro país (Discoli, 2009).

\subsection{Etapas para la construcción de escenarios urbano-energéticos}

Los escenarios urbano-energéticos propuestos están relacionados con variables socio-demográficas, morfológicas urbanas, constructivas y de equipamiento domiciliario, las cuales inciden sobre el consumo energético. Su elaboración consta de tres etapas, que se describen a continuación y se sintetizan en la figura 1. 


\section{Etapa 1: Base (situación de referencia)}

E 1.1. Analizar cualitativa y cuantitativamente el contexto político, económico, socio-demográfico y energético del área de estudio, tanto en el presente como sus trayectorias previas.

E 1.2. Normalizar y sistematizar, en una base de datos, información desagregada que incluya variables e indicadores socio-demográficos, morfológicos urbanos, tecnológico-constructivos, de equipamiento domiciliario y de demanda/oferta de energía, utilizando diferentes niveles de análisis, escalas territoriales y cortes temporales, enfatizando en el sector residencial discriminado por áreas homogéneas urbano-energéticas (AH).

E 1.3. Definir y construir el "año base”, respecto del cual se evaluará el comportamiento de las principales variables que analizar en un determinado año, focalizando en los diferentes sectores urbanos. En este caso, se utiliza el software LEAP, que permite modelar sistemas energéticos integrales y construir escenarios.

\section{Etapa 2: Escenarios "tendencial” y "eficiente"}

E 2.1. Elaborar las proyecciones socio-demográficas que manifiesten el desarrollo del crecimiento poblacional, de viviendas y económico. Estas proyecciones serán comunes para los diferentes escenarios energéticos.

E 2.2. Construir un escenario urbano-energético "tendencial", que examine los cambios probables en la demanda y en la oferta energética para los años siguientes manteniendo las trayectorias observadas.

E 2.3. Construir, ensayar y evaluar escenarios urbano-energéticos "eficientes" con diferentes combinaciones de medidas de eficiencia energética y energías renovables en la demanda y en la oferta de energía.

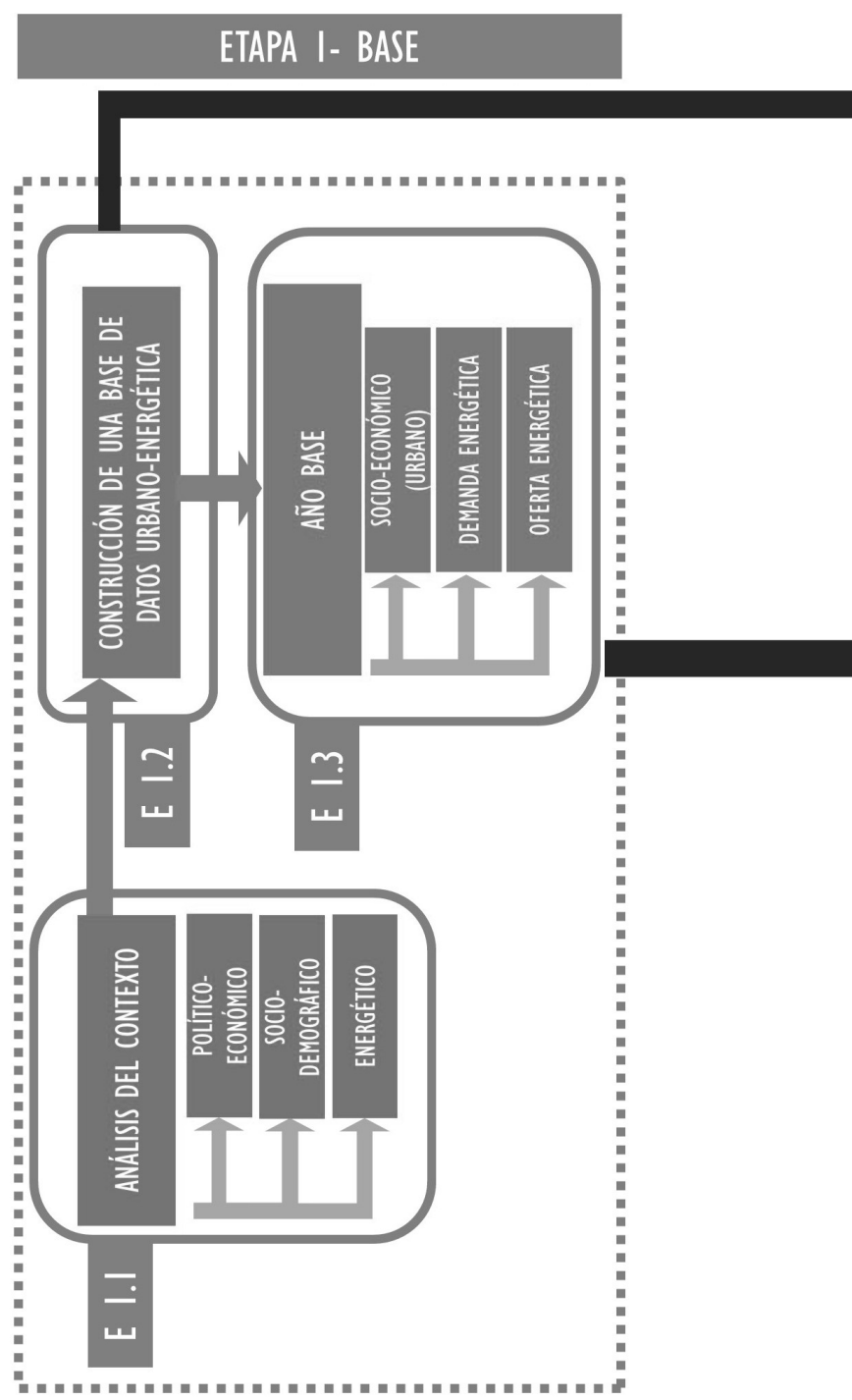




\section{Desarrollo metodológico para la construcción de escenarios urbano-energéticos de largo plazo}

\section{ETAPA 2- ESCENARIOS}

ETAPA 3- EVALUACIÓN

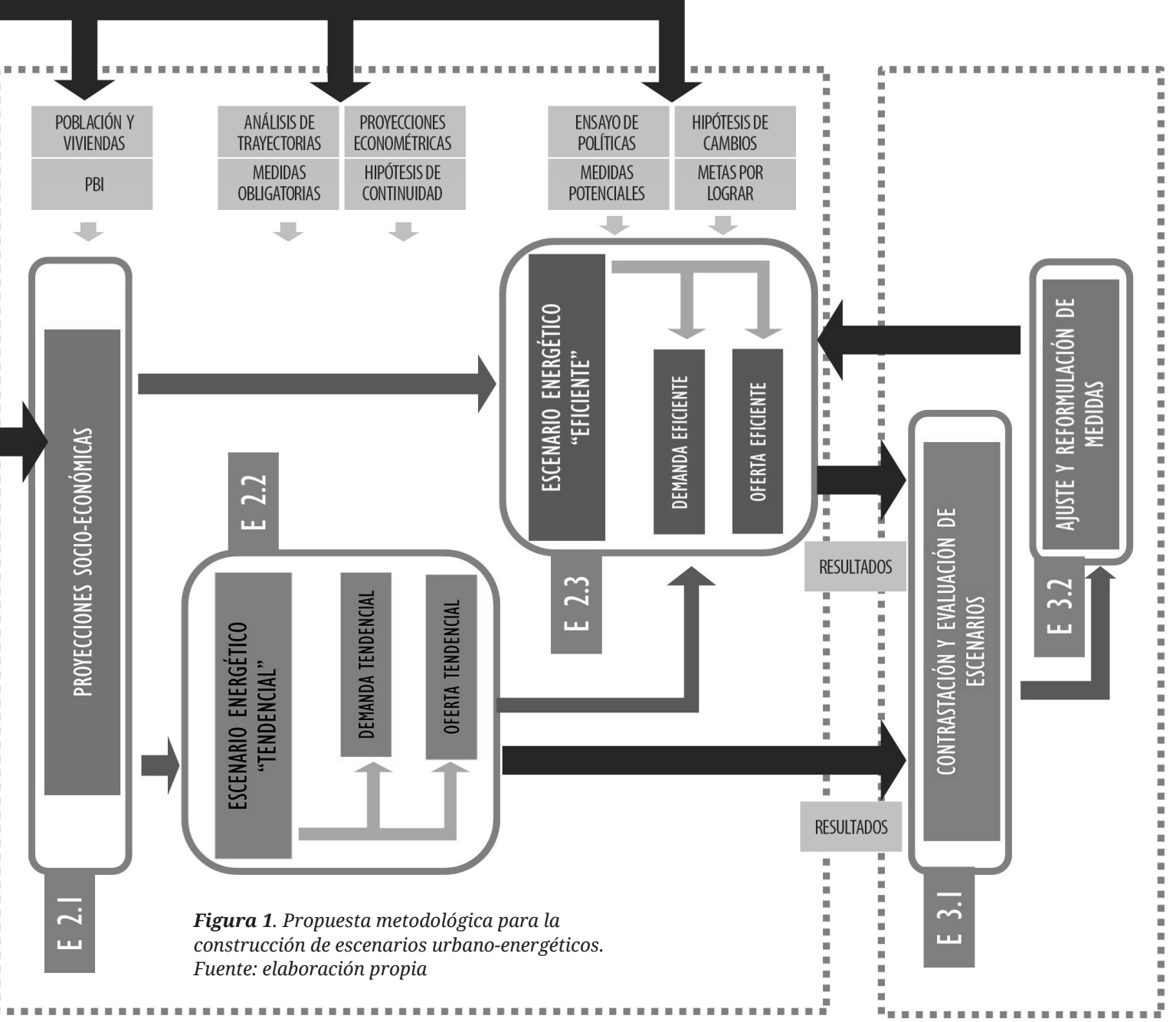




\section{Etapa 3: Evaluación}

E 3.1. Realizar una comparativa de los escenarios urbano-energéticos construidos, a partir de indicadores que permitan evaluar su impacto en aspectos como el ambiente, costos, sustitución de combustibles fósiles, habitabilidad y otros.

E 3.2. Evaluar el impacto de las medidas propuestas en el escenario "eficiente", reformular el alcance e iterar el proceso.

Teniendo en cuenta el objeto de estudio y la metodología propuesta, la cual incluye tanto la demanda como la oferta energética y profundiza el análisis sobre el sector residencial, es preciso utilizar diferentes niveles de análisis (global, sectorial, detallado), comprendidos por distintas escalas territoriales (urbana, áreas homogéneas, mosaicos urbanos y viviendas). Esto permite sistematizar el relevamiento de información de diversa procedencia y de manera desagregada.

A continuación se describen los niveles y escalas, las cuales se sintetizan en la figura 2 .

\subsection{Abordaje del universo de análisis}

\section{Nivel de análisis global (NAG)}

El análisis global, en primer lugar, analiza la estructura urbana; todos los sectores de consumo (residencial, comercial, industrial, servicios generales, oficial, transporte, alumbrado público, etc.) y el abastecimiento de la oferta energética a escala urbana $\left(\mathrm{NAG}_{\mathrm{URB}}\right)$. Luego, en un nivel inferior releva la información del sector residencial $\left(\mathrm{NAG}_{\mathrm{RES}}\right)$ y evalúa el comportamiento de diversas variables socio-demográficas y energéticas en el territorio.

\section{Nivel de análisis sectorial (NAS)}

Este nivel de análisis aborda y se circunscribe a las áreas homogéneas urbano-energéticas residenciales $(\mathrm{AH})$ que se agrupan según su similitud interna respecto de una serie de variables socio-demográficas y energéticas predefinidas. 


\section{Desarrollo metodológico para la construcción de escenarios urbano-energéticos de largo plazo}

\section{Figura 2 METODOLOGIÁ DE ABORDAIE PARA DEL OBJETO DE ESTUDIO. NIVELES DE ANÁLISIS E INTEGRACIÓN DE LA INFORMACIÓN.}
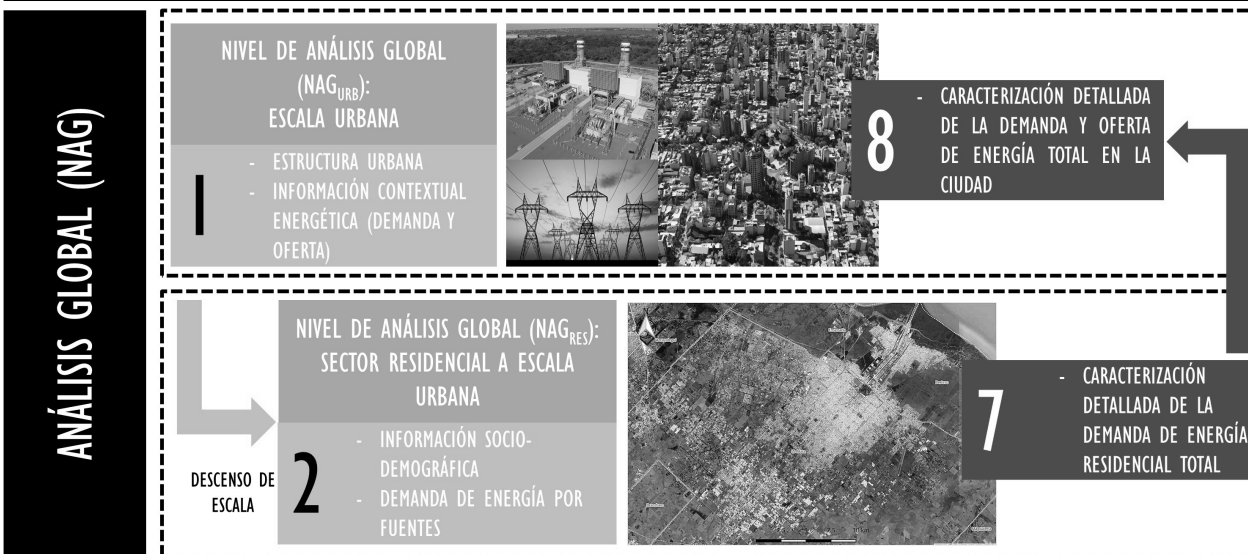

INTEGRACIÓN DE LA INFORMACIÓN
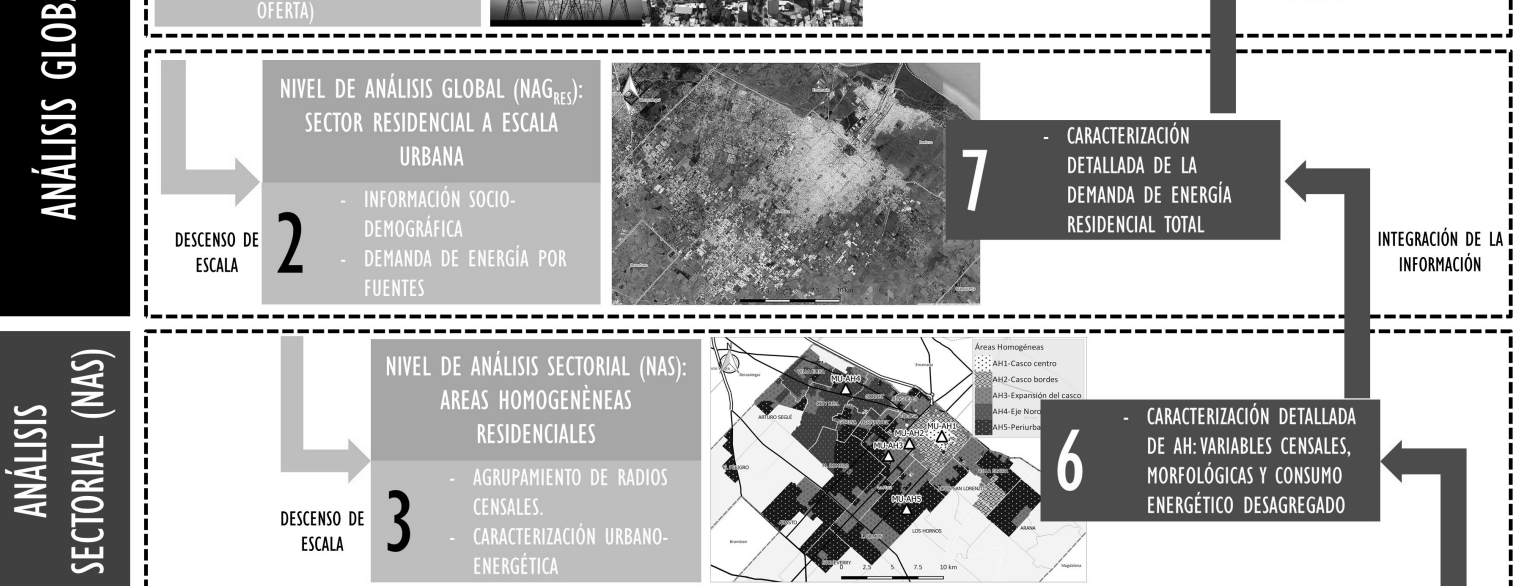

CARACTERIZACIÓN DETALLAD

DE AH: VARIABLES CENSALES,

MORFOLÓGICAS Y CONSUMO ENERGÉTICO DESAGREGADO
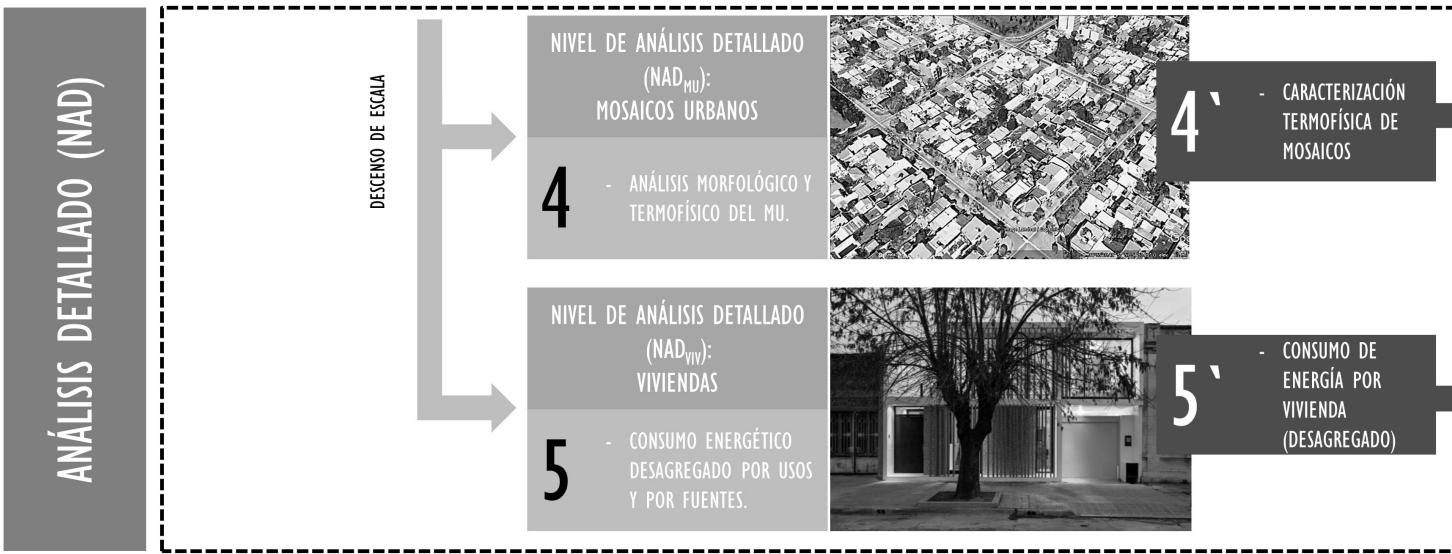

Abordaje del universo de análisis. Fuente: elaboración propia 


\section{Nivel de análisis detallado (NAD)}

El análisis detallado incluye dos escalas territoriales. Para cada una de las áreas homogéneas se analizan, de forma paralela, un mosaico urbano $\left(\mathrm{NAD}_{\mathrm{MU}}\right)$ y una muestra estadística de viviendas $\left(\mathrm{NAD}_{\text {viv }}\right)$, en ambos casos representativos del área.

Una vez abordada la descripción del caso de estudio, las etapas generales y el método de aproximación al universo de análisis, a continuación se presenta el desarrollo metodológico para la construcción de los escenarios urbano-energéticos. Para ello se expone, inicialmente, la metodología para la construcción del "año base" (sección 3); luego, el desarrollo para la construcción de los escenarios alternativos — "tendencial” y "eficiente" - (sección 4) y, finalmente, la evaluación y comparativa de estos (sección 5).

\section{Metodología para la construcción del "año base”}

El "año base" sintetiza el comportamiento de las principales variables que analizar en un determinado año (2014 en el presente trabajo). Se utiliza como año inicial en la simulación de escenarios y se construye a partir de información urbano-energética sintetizada en una base de datos que contiene variables/indicadores socio-demográficos, morfológicos urbanos, constructivos, como así también de oferta y demanda de energía.

En primera instancia se realiza un relevamiento "en cascada", comenzando el análisis por el nivel global hasta alcanzar el detallado, cada uno con técnicas de relevamiento y procesamiento específicas y propias de cada escala territorial. Esta primera etapa se observa a la izquierda de la figura 2. En segundo lugar, la información obtenida y procesada en cada escala de trabajo es integrada hacia los niveles superiores, lo cual permite una caracterización y descripción exhaustivas del objeto de estudio. Esta segunda etapa se observa a la derecha en la figura 2. Los métodos, técnicas y variables utilizados en cada nivel de análisis para la construcción del "año base" (AB) se sintetizan a continuación. 


\section{AB. Nivel de análisis global}

En el nivel de análisis global a escala urbana $\left(\mathrm{NAG}_{\mathrm{URB}}\right)$ se evalúan los siguientes aspectos. Inicialmente, se analizan brevemente tres elementos de la estructura urbana: el soporte natural (clima y topografía), el soporte físico (trazado urbano, servicios, actividades económicas, etc.) y el soporte socio-demográfico (caracterización de población y de los hogares). De esta manera, se cuenta con información contextual que contribuye a detectar patrones de consumo de energía propios del caso de estudio.

Posteriormente, se releva información contextual energética del caso de estudio, es decir, la demanda de energía (por fuentes y por sectores de consumo) y la oferta de energía. La figura 3 sintetiza el sistema energético modelado para la ciudad de La Plata, el cual incluye el abastecimiento y la demanda de energía con los valores energéticos correspondientes. El sector de la demanda fue dividido en cuatro secciones: CISGyO (comercial, industrial, servicios generales y oficial), alumbrado público, transporte y residencial (dividido en cinco áreas homogéneas urbanas, AH). La oferta energética fue modelada en escala, en función de los valores reales que presentan los distintos sistemas nacionales (figura 3 ).

En el nivel de análisis global del sector residencial $\left(\mathrm{NAG}_{\mathrm{RES}}\right.$ ), se evalúan los siguientes aspectos: en primer lugar, se realiza un análisis territorial de las características urbano-energéticas del sector residencial, donde se requiere evaluar información territorial socio-demográfica. Para ello, se estudia individualmente el comportamiento de once variables en el territorio, representadas a nivel de radio censal. La información es extraída del censo nacional 2010 y se procesa en un soporte GIS. Las variables seleccionadas son: 1. personas/hogar; 2. habitaciones/hogar; 3. hogares con necesidades básicas insatisfechas; 4. hogares con gas por red; 5. hogares con tipología de vivienda 'casa'; 6 . hogares con vivienda de calidad constructiva 'satisfactoria'; 7.habitantes/hectárea; 8. hogares/hectárea; 9. personas ocupadas; 10. edad promedio y 11. hogares con vivienda propia. 


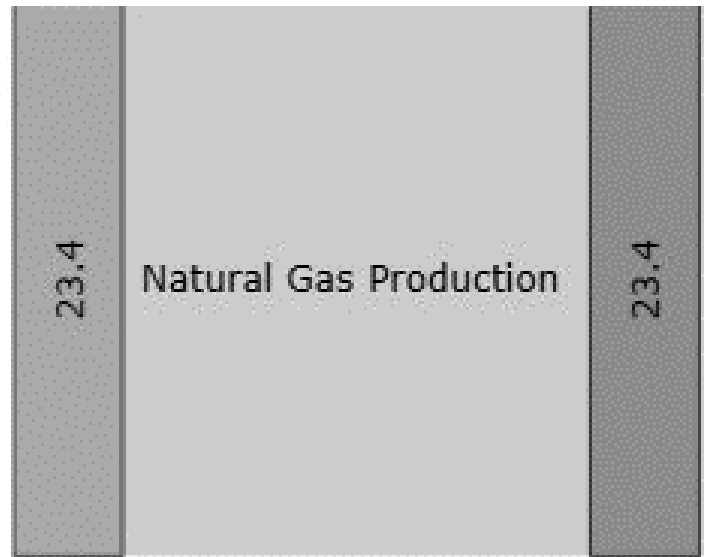

\section{Plantas de tratamiento de gas}

Pérdidas de gas distribuido por redes

$\frac{4}{\mathrm{~N}}$

i Hydro Production

$\stackrel{\infty}{\circ}$ Nuclear Production

m Coal Bituminous Production

$\stackrel{-1}{0}$ Wind Production

응 Solar Production

$\stackrel{-1}{\circ}$ Wood Production
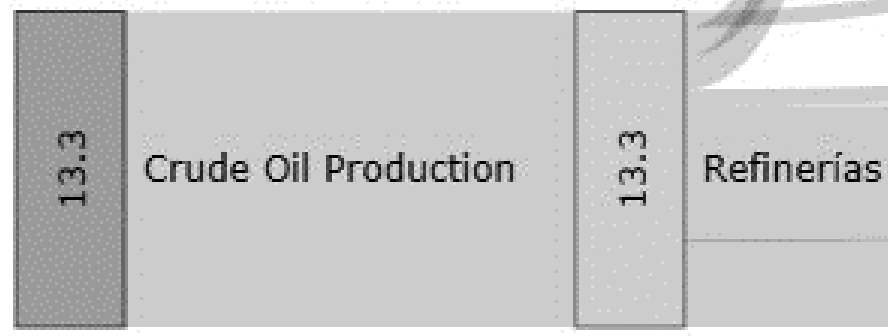

Figura 3. Agrupamiento de la demanda de energía en sectores de consumo. Fuente: elaboración propia 


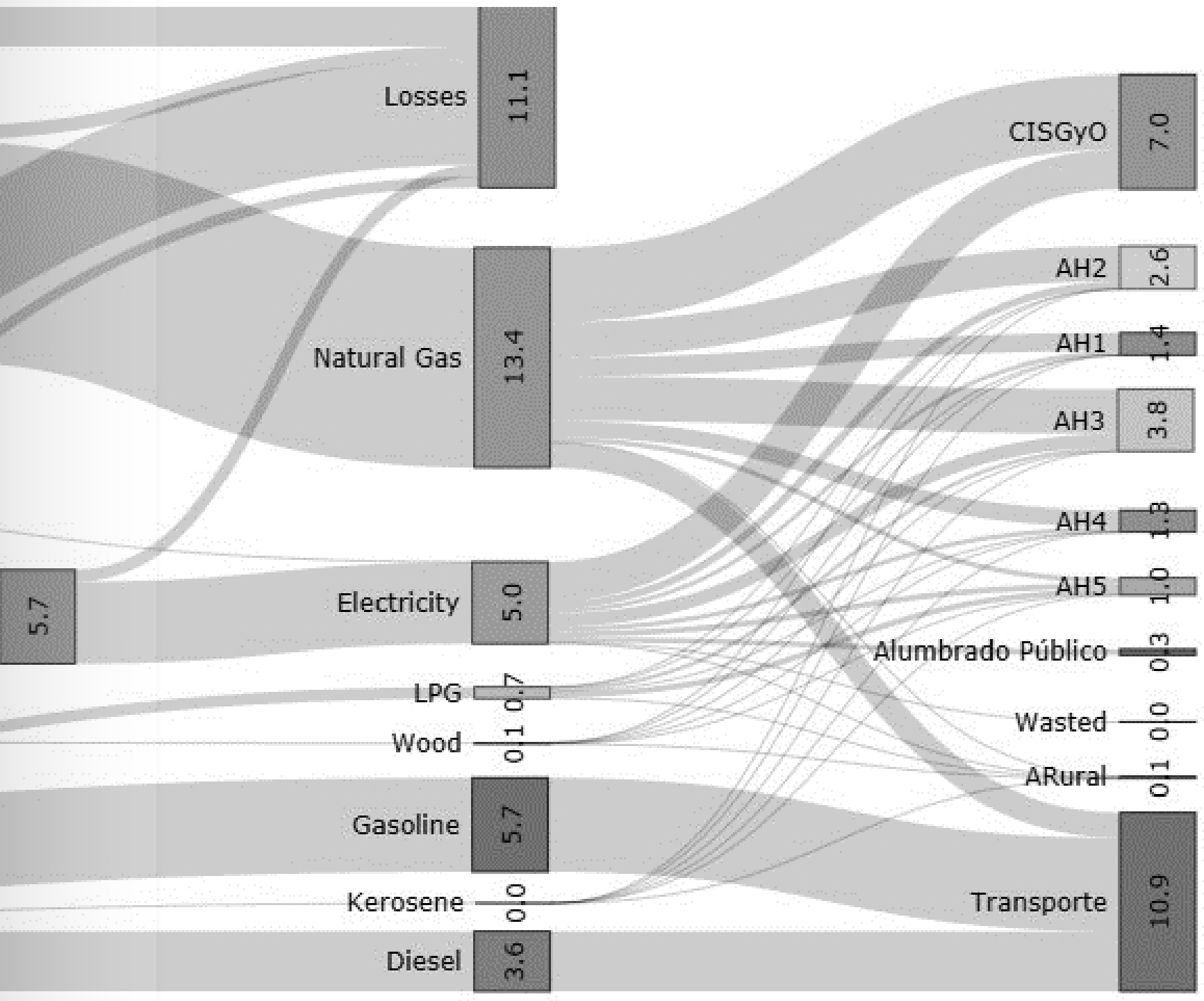


Posteriormente se releva la información territorial energética, en la cual se analiza el consumo de tres vectores energéticos por usuario en todo el territorio, también desagregados a nivel de radio censal: 1 . electricidad, 2 . gas natural y 3 . combustibles a granel.

En última instancia se analizan las correlaciones entre variables socio-demográficas y de consumo energético residencial, a los efectos de identificar cuáles presentan mayores niveles de $\mathrm{R}^{2}$. Para ello, se utiliza la información asociada a los radios censales y se elaboran gráficos de dispersión entre cada variable socio-demográfica y los tres vectores energéticos, los cuales son sintetizados en fichas temáticas. La figura 4 presenta la ficha temática de la variable 7. habitantes/hectárea, donde se observa el mapa correspondiente, una breve descripción estadística de la variable y el cruce con el consumo de los tres vectores energéticos (figura 4).

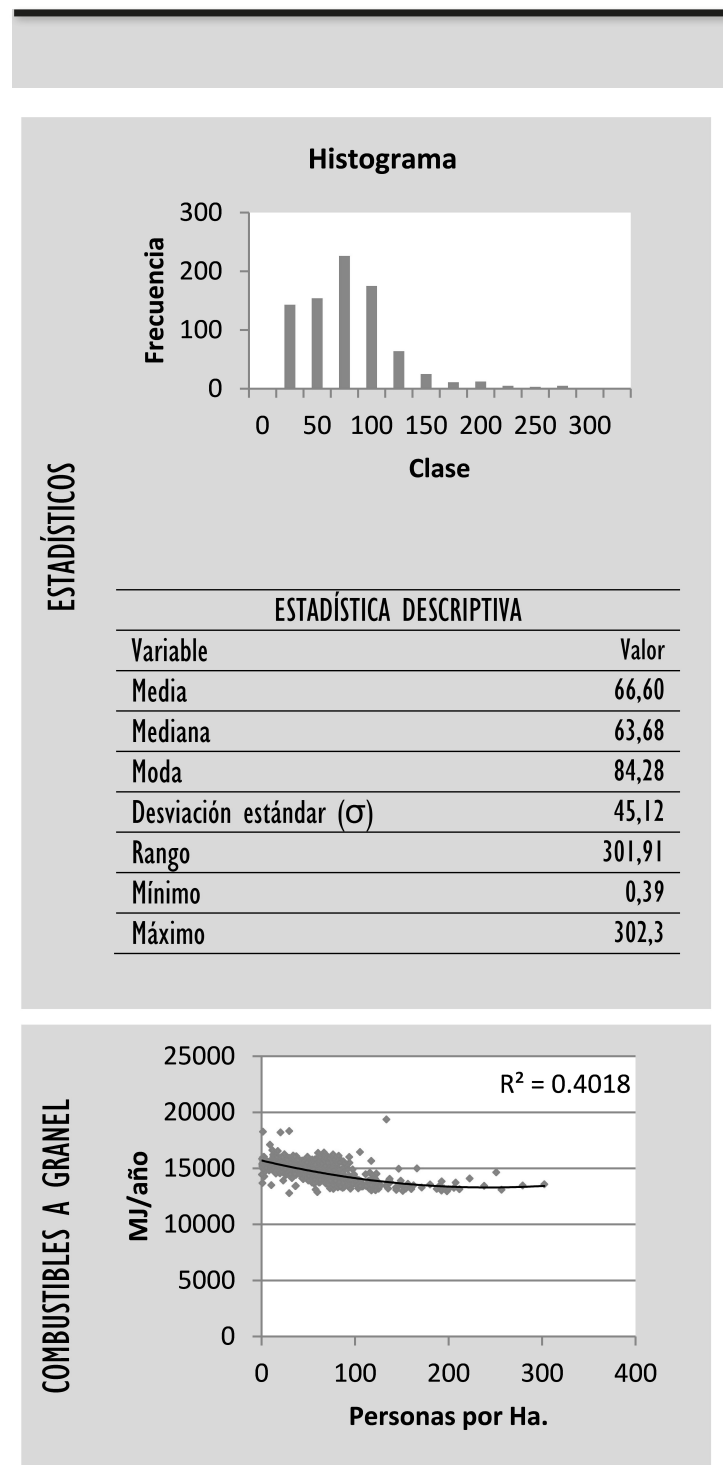




\section{Desarrollo metodológico para la construcción de escenarios urbano-energéticos de largo plazo}

7.- DENSIDAD POBLACIONAL: CANTIDAD DE HABITANTES POR HECTÁREA POR RADIO CENSAL (Escala I:400.000)
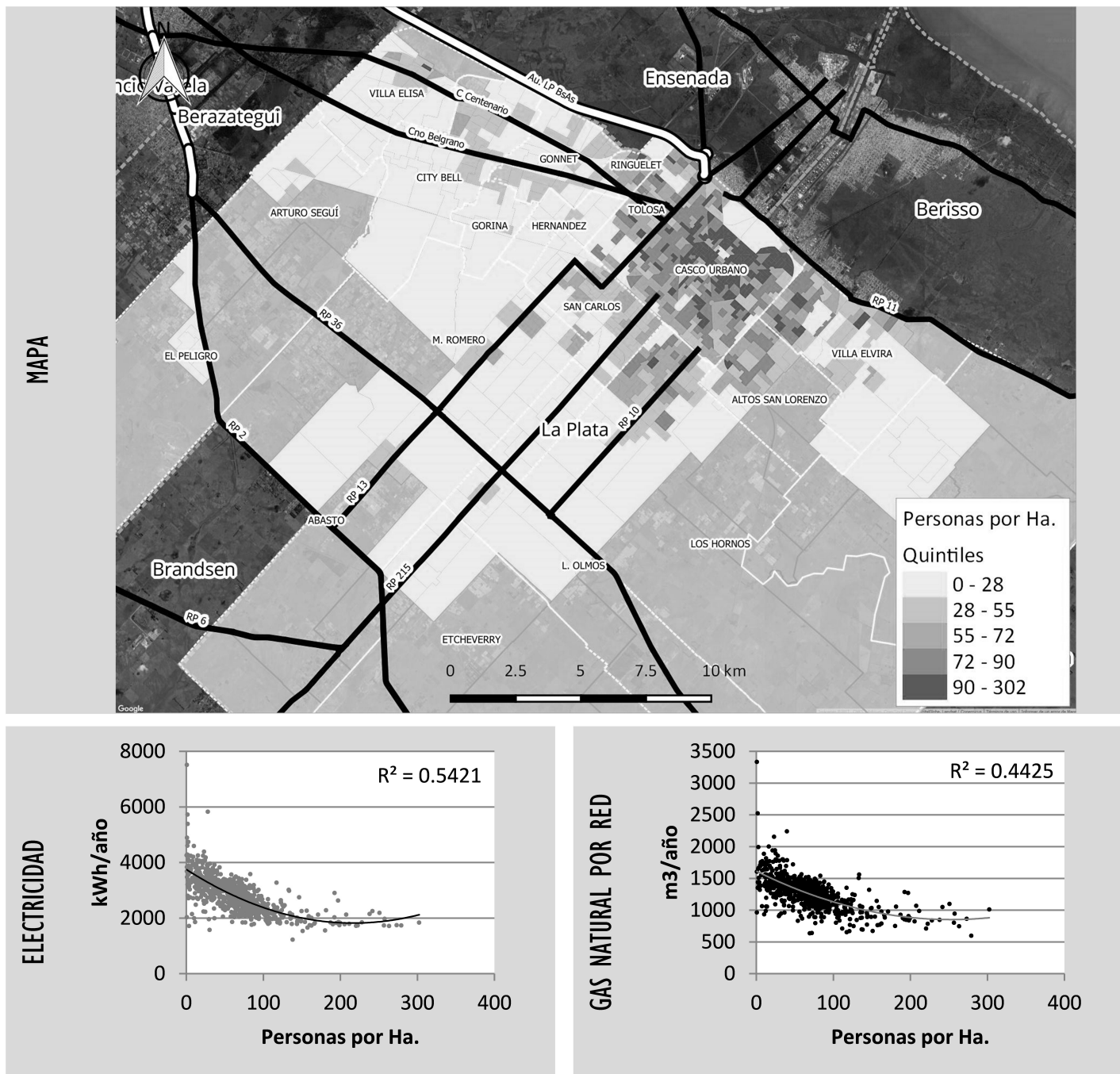

Figura 4.

Ejemplo de la ficha temática 7-Habitantes/ hectárea. Fuente: elaboración propia 
AB. Nivel de análisis sectorial: el NAS está constituido por las áreas homogéneas urbano-energéticas. Para su delimitación se opta por el agrupamiento de radios censales (RC) que presenten características similares en cuanto a las variables identificadas como clave en el nivel $\mathrm{NAG}_{\mathrm{RES}}$, es decir, las variables energéticas y aquellas socio-demográficas con mayor grado de correlación. Para el caso de estudio de La Plata, las variables seleccionadas resultaron: personas/hogar, habitaciones/hogar, hogares con tipología de vivienda 'casa'; hogares/hectárea; consumo promedio de electricidad [kWh/año por usuario] y consumo promedio de gas natural [ $\mathrm{m}^{3} / \mathrm{año}$ por usuario]. Una vez seleccionadas las variables, se utiliza la técnica de clustering de k-medias para agrupar vectores. Cada uno de los " $n$ " casos que agrupar (" $n$ " = 825 radios censales) cuenta con un vector de "d" dimensiones ("d" = 6 variables), con sus valores normalizados entre 0 y 1, los cuales se agrupan según

Figura $5 a$. Imagen satelital.

Fuente:

elaboración propia, sobre la base de Google Maps

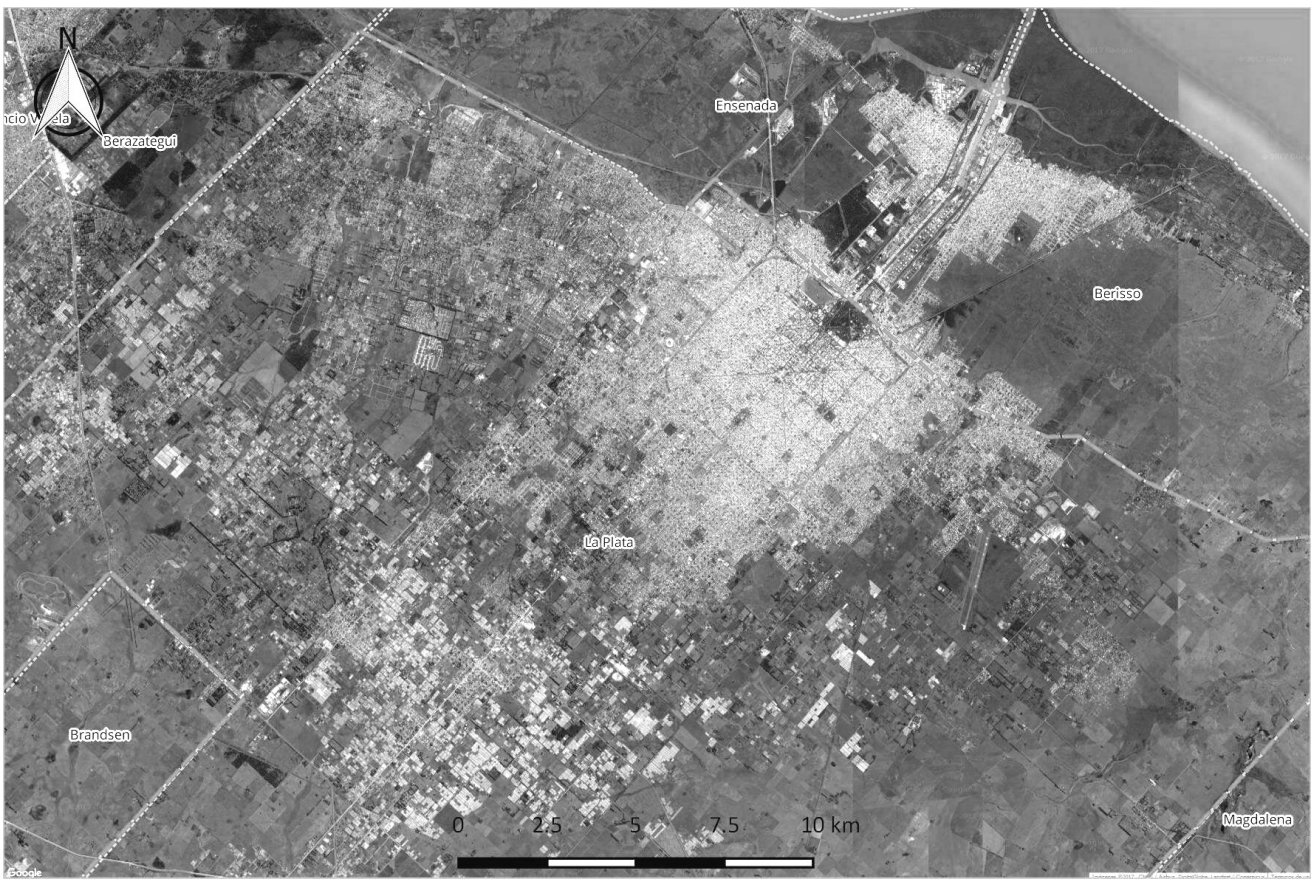




\section{Desarrollo metodológico para la construcción de escenarios urbano-energéticos de largo plazo}

su nivel de afinidad en "k" clusters (" $k$ " 5 clusters). La cantidad de clusters se debe ensayar según las características del caso de estudio y, en función de los diversos resultados, se requiere seleccionar aquella configuración que identifique con el menor número de grupos posible las heterogeneidades de la ciudad. Cada uno de los clusters conforma lo que se denominan áreas-homogéneas, las cuales tienen un comportamiento interno similar respecto de las variables seleccionadas. La figura 5 muestra el resultado del clustering aplicado en el caso de estudio.

Una vez delimitadas las áreas homogéneas, se requiere el análisis de la información asociada a cada una de estas, para lo cual en este nivel de análisis es posible relevar datos censales y totalizar el consumo energético de cada área, discriminado por fuentes.

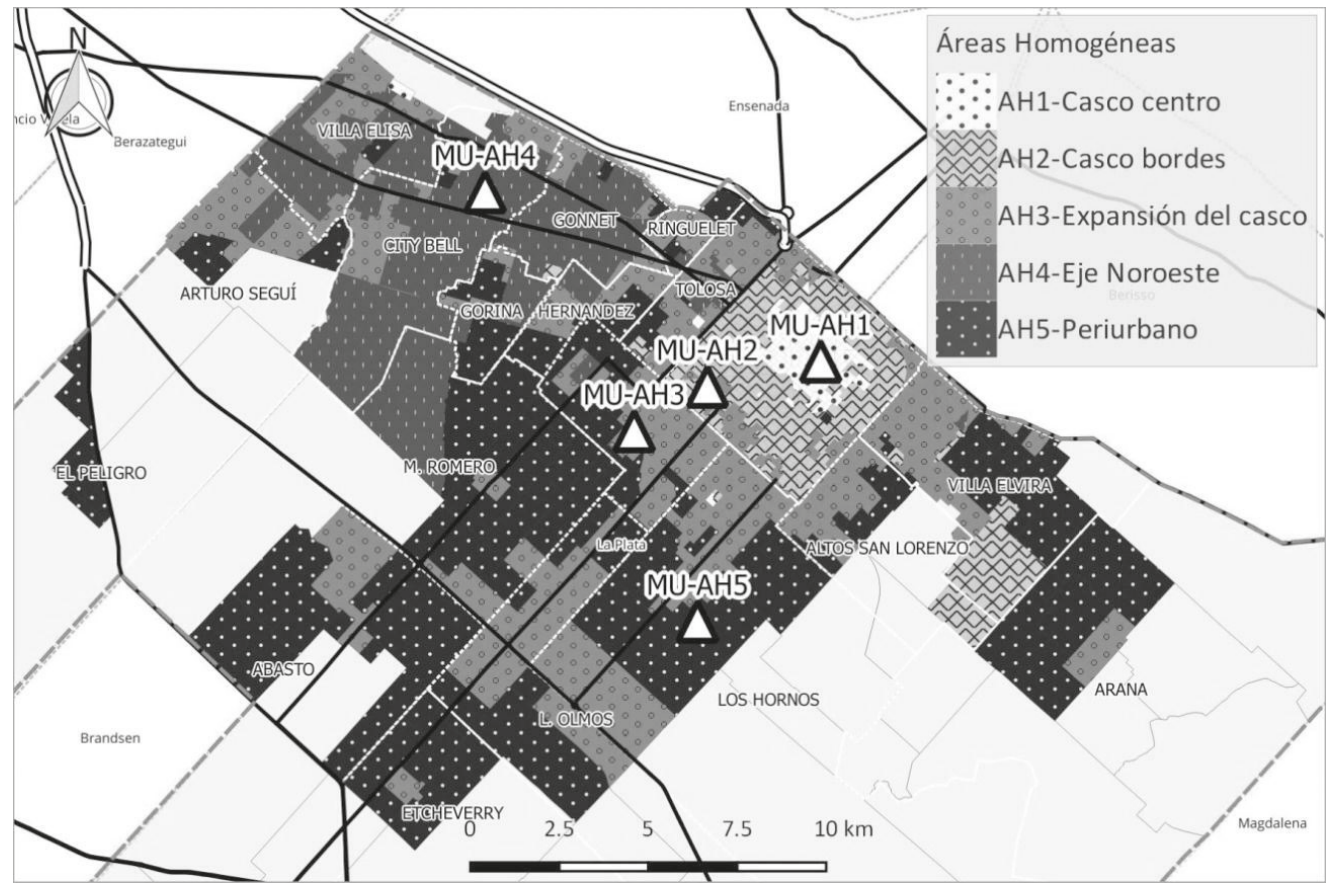

Figura $5 b$

Delimitación de las AH. Escala: 1.450 .000 .

Fuente: elaboración propia 
AB. Nivel de análisis detallado: en el nivel de análisis detallado, conformado por los mosaicos urbanos $\left(\mathrm{NAD}_{\mathrm{MU}}\right.$ ), se realiza una caracterización morfológica y termofísica de las áreas homogéneas. Los MU son pequeños sectores de la ciudad, representativos de áreas mayores. Estos permiten el estudio de grandes áreas sin necesidad de efectuar un relevamiento sobre toda su extensión. Su análisis se realiza a partir de fotografías aéreas, relevamiento de campo y representación gráfica en dos y tres dimensiones.

Para cada una de las AH se selecciona un MU para ser analizado, se obtienen superficies de envolvente, nivel de asoleamiento de muros y techos, superficies construidas, entre otras. A su vez, se calcula el coeficiente de transmitancia térmica ponderada de la envolvente ( $\mathrm{K}_{\text {medio }}-\mathrm{W} / \mathrm{m}^{2} \mathrm{~K}$-) y el coeficiente volumétrico de pérdidas $\left(\mathrm{G}_{\mathrm{cal}}-\mathrm{W} / \mathrm{m}^{3} \mathrm{~K}-\right.$ ). Este se contrasta con el máximo admisible establecido por las regulaciones $\left(G_{a d m}-W /\right.$ $\mathrm{m}^{3} \mathrm{~K}-$ ), a los efectos de conocer el potencial de ahorro en la demanda energética de calefacción. Asimismo, se efectúan mediciones de temperatura exterior para calcular los grados día (GD) de cada sector (VIEGAS ET ÁL., 2017). Este relevamiento permite conocer el potencial de incorporación de sistemas de energías renovables y el mejoramiento de la envolvente edilicia. Para el caso de estudio de la ciudad de La Plata, se seleccionaron los mosaicos que se presentan en la tabla 1.

Una vez seleccionados los MU representativos de cada área, se requiere la verificación de la representatividad estadística dentro de ella. Para esto es necesario analizar la distancia euclídea simple del vector utilizado para el clustering del radio censal donde se ubica el MU hasta el vector centroide del AH. La tabla 1 sintetiza dicha información e incluye un histograma de frecuencias de las distancias euclídeas de los RC que conforman cada $\mathrm{AH}$.

En el nivel de análisis detallado, que está conformado por la muestra estadística de viviendas $\left(\mathrm{NAD}_{\mathrm{VIV}}\right)$, se realiza una caracterización individual a partir de encuestas energéticas en las que se releva información del consumo desagregado por usos. Para ello, se necesita el diseño de una encuesta específica para el caso de estudio, dado que la utilización de las fuentes energéticas difiere según sea el caso de estudio. A su vez, es preciso determinar el tipo de encuesta (presencial /en línea / telefónica), las preguntas, las expresiones de cálculo y también las herramientas necesarias para el procesamiento de la información, entre otros aspectos. 


\section{Desarrollo metodológico para la construcción de escenarios urbano-energéticos de largo plazo}

\begin{tabular}{|c|c|c|c|}
\hline & Imagen & $\begin{array}{l}\text { Histograma de } \\
\text { frecuencias de } \\
\text { distancias de RC }\end{array}$ & $\begin{array}{c}\text { Distancia del } \\
\mathrm{RC} \text { al centroide } \\
\text { del AH }\end{array}$ \\
\hline MU-AH1 & & 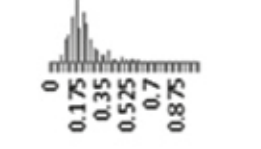 & 0,25 \\
\hline MU-AH2 & & 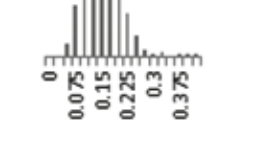 & 0,13 \\
\hline MU-AH3 & & 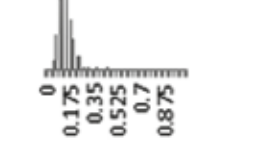 & 0,08 \\
\hline MU-AH4 & int & 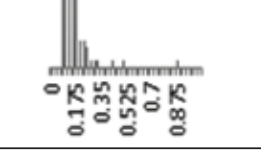 & 0,069 \\
\hline MU-AH5 & & 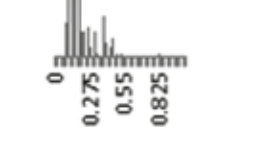 & 0,37 \\
\hline
\end{tabular}


Por su parte, se debe determinar la muestra que encuestar en cada área homogénea, cuya expresión debe contar con sustento estadístico. Para ello, se adopta como variable para el cálculo de la muestra el consumo eléctrico por usuario, puesto que es el único servicio energético que alcanza a la totalidad de las viviendas e involucra a la mayor cantidad de equipos domiciliarios. Dicha variable manifiesta algunas características del sector de estudio, como la presencia del gas por red o ciertas condiciones socio-demográficas. Una vez seleccionada la variable, se aplica el método para determinar muestras estratificadas a partir de afijación proporcional sin reposición (PÉREz LóPez, 2005). La ecuación 1 calcula la muestra total de casos que encuestar, para lo cual se necesita la provisión de datos por parte de la distribuidora eléctrica:

$$
n=\frac{\sum_{h=1}^{L} W_{h} S_{h}^{2}}{e^{2}+\frac{1}{N} \sum_{h=1}^{L} W_{h} S_{h}^{2}}
$$

Donde

$\mathrm{n}=$ tamaño de la muestra total a estratificar

$\mathrm{W}_{h}=\frac{\mathrm{N}_{h}}{\mathrm{~N}}=\begin{gathered}\text { cociente entre el tamaño del estrato } \mathrm{h} \text { (usuarios en un } \mathrm{AH} \text { ) } \\ \text { y el universo de análisis (total usuario en la ciudad) }\end{gathered}$

e= error máximo permitido

$\mathrm{N}=$ tamaño del universo de análisis (total usuarios en la ciudad)

$\mathrm{S}_{h} 2=$ cuasivarianza poblacional en el estrato $\mathrm{h}$. La misma se calcula a partir de la siguiente expresión (Ec. 2):

$$
S_{h}^{2}=N_{h} \frac{\sigma_{h}^{2}}{\left(N_{h}-1\right)}
$$


Donde:

$\mathrm{N}_{h}=$ tamaño del estrato $\mathrm{h}$ (usuarios en un $\mathrm{AH}$ )

$\sigma_{h}^{2}=$ varianza del estrato $h$ (varianza del consumo eléctrico promedio anual en un $\mathrm{AH}$ )

Una vez hallado el tamaño total de la muestra, se realiza la afijación proporcional sin reposición de cada uno de los estratos con la Ecuación 3:

$$
n_{h}=k N_{h} \quad \text { con } k=\frac{n}{N} \quad \begin{aligned}
& n_{1}=k N_{1} \\
& n_{2}=k N_{2} \\
& n_{h}=k N_{h}
\end{aligned}
$$

A partir del relevamiento y procesamiento de la encuesta, se desagrega el consumo residencial en las tres fuentes energéticas (electricidad, gas natural y combustibles a granel) en los siguientes usos (grupos de consumo):

-Electricidad: [1] Climatización (1.1. Efecto Joule y ventiladores, 1.2.- $\mathrm{A}^{\circ} \mathrm{A}^{\circ}$ individual, 1.3. $\mathrm{A}^{\circ} \mathrm{A}^{\circ}$ central); [2] Cocción; [3] Agua caliente sanitaria; [4] Refrigeración de alimentos; [5] Iluminación; [6] Lavado; [7] Informática y celulares; [8] Televisión, audio y video y [9] Otros electrodomésticos.

-Gas natural por red: [1] Climatización; [2] Cocción y [3] Agua caliente sanitaria.

-Combustibles a granel: [1] Climatización; [2] Cocción y [3] Agua caliente sanitaria.

AB. Integración de la información: en primer lugar, se realiza una integración en el nivel sectorial — por AH- (NAS); para ello, se elaboró un programa basado en Visual Basic y ejecutado mediante macros en el software Excel — denominado CC-Área-, que permite analizar la información detallada de las distintas AH, e incluso de la totalidad de la ciudad, en un único archivo. El programa permite seleccionar cada una de las AH, analizar sus características socio-demográficas, morfológicas-urbanas y el consumo desagregado por fuentes y por usos (figura 6). Este análisis ayuda a comprender la potencialidad de las distintas áreas homogéneas para la incorporación de estrategias de EE y ER y, a su vez, direccionar medidas tendientes a disminuir los consumos más importantes de cada sector. 


\section{SINTESIS DEL CONSUMO ENERGÉTICO DESAGREGADO POR USOS}

\begin{tabular}{|c|c|c|c|c|c|c|c|c|c|c|c|c|c|c|c|c|}
\hline \multicolumn{2}{|l|}{ Área Homogénea } & \multicolumn{15}{|l|}{2} \\
\hline \multirow[b]{2}{*}{ Grupos de consumo eléctrico } & \multicolumn{5}{|c|}{ "Año base" } & \multicolumn{5}{|c|}{ Escenario "tendencial" } & \multicolumn{6}{|c|}{ Escenario "eficiente" } \\
\hline & 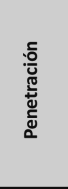 & 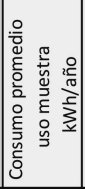 & 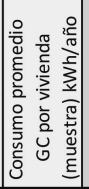 & 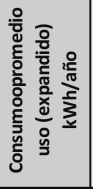 & 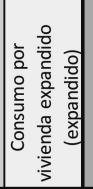 & 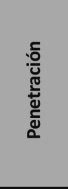 & 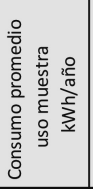 & 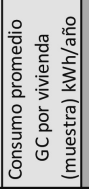 & 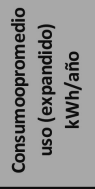 & 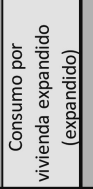 & 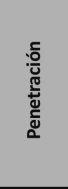 & 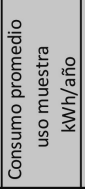 & 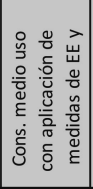 & 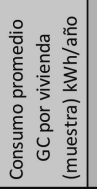 & 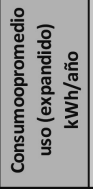 & 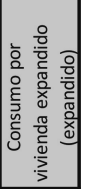 \\
\hline 1.1-Climatización 1.- $\mathrm{A}^{\circ} \mathrm{A}^{\circ} \mathrm{E}$. Joule y Vent. & 0.894 & 346.0 & 309 & 285.83 & 256 & 0.925 & 438.62 & 406 & 368.49 & 341 & 0.925 & 438.6 & 286.9 & 265 & 241.00 & 223 \\
\hline 1.2-Climatización 2.- $\mathrm{A}^{\circ} \mathrm{A}^{\circ}$ individual & 0.627 & 489.7 & 307 & 404.49 & 254 & 1.160 & 416.21 & 483 & 349.66 & 405 & 1.160 & 391.7 & 278.4 & 323 & 233.92 & 271 \\
\hline 1.3-Climatización 3.- $\mathrm{A}^{\circ} \mathrm{A}^{\circ}$ central & 0.028 & 2010.9 & 57 & 1661.18 & 47 & 0.051 & 2010.94 & 102 & 1689.42 & 85 & 0.051 & 1608.8 & 1052.2 & 81 & 1351.54 & 68 \\
\hline 2-Cocción & 0.634 & 124.0 & 79 & 102.43 & 65 & 0.821 & 124.00 & 102 & 104.17 & 86 & 0.821 & 105.4 & & 87 & 88.55 & 73 \\
\hline 3-Agua caliente sanitaria & 0.042 & 1551.3 & 66 & 1281.44 & 54 & 0.042 & 1551.25 & 66 & 1303.23 & 55 & 0.042 & 1225.5 & 818.1 & 35 & 687.33 & 29 \\
\hline 4-Refrigeración de alimentos & 1.190 & 520.5 & 619 & 429.96 & 512 & 1.396 & 397.22 & 554 & 333.71 & 466 & 1.396 & 259.9 & & 363 & 218.36 & 305 \\
\hline 5-Iluminación & 6.507 & 57.6 & 375 & 47.61 & 310 & 8.265 & 50.16 & 415 & 42.14 & 348 & 8.265 & 29.4 & & 243 & 24.67 & 204 \\
\hline 6-Lavado de ropa & 1.092 & 201.2 & 220 & 166.22 & 181 & 1.316 & 171.49 & 226 & 144.07 & 190 & 1.316 & 118.6 & & 156 & 99.66 & 131 \\
\hline 7-Informática y celulares & 3.169 & 157.3 & 499 & 129.98 & 412 & 3.690 & 150.77 & 556 & 126.67 & 467 & 3.690 & 135.0 & & 498 & 113.45 & 419 \\
\hline 8-Televisión, audio y video & 2.296 & 87.9 & 202 & 72.59 & 167 & 1.658 & 108.28 & 179 & 90.96 & 151 & 1.658 & 49.1 & & 81 & 41.27 & 68 \\
\hline 9-Otros electrodomésticos & 0.958 & 246.4 & 236 & 203.54 & 195 & 0.958 & 492.79 & 472 & 414.00 & 397 & 0.958 & 443.5 & & 425 & 372.60 & 357 \\
\hline Generación fotovoltaica & 0.000 & 0.0 & 0 & 0.00 & 0 & & & 0 & 0.00 & 0 & & & & -219 & 0.00 & -219 \\
\hline Total & & & 2968 & & 2451 & & & 3560 & & 2991 & & & & 2557 & & 1929 \\
\hline
\end{tabular}

Figura 6. Síntesis del consumo energético desagregado por usos del Área Homogénea 2: pantalla de la planilla de cálculo del programa "CC-Área". Fuente: elaboración propia
Luego, se integra la información en el nivel global $\left(\mathrm{NAG}_{\mathrm{RES}}\right.$ y $\mathrm{NAG}_{\mathrm{URB}}$ ), en el cual los resultados obtenidos son introducidos en el software LEAP. Allí también se modela la demanda de los distintos sectores de consumo no residenciales utilizando el indicador energía/habitante (MJ/año*hab.); mientras que la demanda energética residencial discriminada para cada $\mathrm{AH}$ se modela según fuentes y usos, utilizando el indicador energía/vivienda (MJ/año*viv.). A su vez, se modela la oferta de energía, en la cual el sistema eléctrico es el que mayor nivel de desagregación presenta.

\section{Metodología para la construcción de escenarios urbano-energéticos}

Con los resultados del "año base” se simulan los escenarios alternativos ("tendencial” y "eficiente”), donde se evalúan las trayectorias de las principales variables en el tiempo, en este caso considerando el período 2015-2040. 


\section{Desarrollo metodológico para la construcción de escenarios urbano-energéticos de largo plazo}

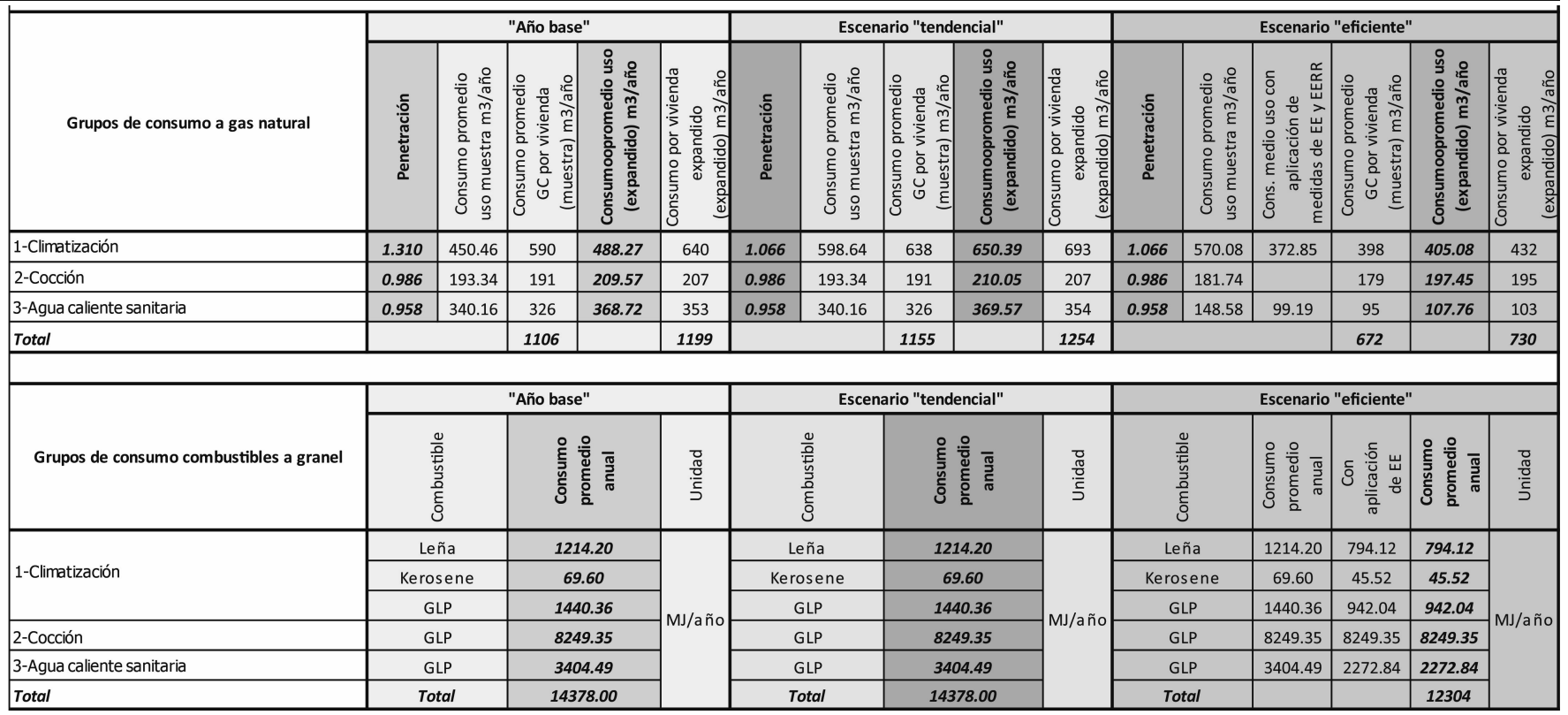

Para su construcción se requiere (i) formular o adoptar proyecciones socio-económicas que permitirán calcular la evolución de la demanda de energía específica y neta del caso de estudio. Luego, se (ii) construye el escenario "tendencial", que permite referenciar la evolución probable de la demanda y la oferta energética para los próximos años en los términos habituales de crecimiento. Finalmente, se (iii) construye el escenario "eficiente", que deriva del "año base" y del escenario "tendencial", donde se evalúan diferentes combinaciones de medidas de EE y ER en el sector de la oferta y la demanda. Una vez que se obtienen los resultados, es posible extraer conclusiones, estudiar el impacto y viabilidad (ambiental, técnica, económica, social y político-institucional) para cada zona analizada, tanto de las medidas como de los escenarios construidos, lo que permite rediseñar y reformular las medidas propuestas. Este hecho ratifica que los escenarios se pueden utilizar como un instrumento de apoyo a la toma de decisiones del planeamiento energético y urbano, en distintas escalas territoriales y horizontes temporales. 
A continuación se profundiza sobre las etapas enunciadas previamente.

\subsection{Relevamiento y elaboración de las proyecciones socio-económicas}

Para la construcción de los escenarios aquí propuestos se requiere relevar o elaborar la proyección de los valores de tres variables socio-económicas: el producto bruto interno (PBI, a nivel nacional), la población (de la ciudad) y las viviendas ocupadas (de la ciudad y por AH). Dichas variables permitirán simular la evolución de la demanda de energía específica y neta del caso de estudio.

En el caso del PBI, este se vincula con los consumos energéticos y permite calcular la demanda específica futura (MJ/año*hab.) de los diferentes sectores a partir de regresiones econométricas. Asimismo, la utilización del PBI como estimador del consumo de energía presenta la ventaja de que diversos organismos elaboran proyecciones de mediano y largo plazo de este indicador, lo que evita el abordaje de un campo disciplinar complejo. En este sentido, se relevó la información provista por el FMI (2016) y OCDE (2012).

En el caso de la evolución de la población del área de estudio, esta se utiliza para calcular la energía neta de los sectores no residenciales - CISGyO, alumbrado público y transporte(MJ/año), la cual surge de multiplicar la demanda de energía específica (MJ/año*hab.) por dicha variable (habitante). Por lo tanto, se utilizan las proyecciones de población elaboradas por la Dirección Provincial de Estadística (DPE, 2016), que llegan hasta 2025, y una regresión matemática que extiende la función aplicada por la DPE para alcanzar el horizonte temporal del año 2040.

En tanto, la evolución de las viviendas ocupadas, proyectadas de forma desagregada por área homogénea, se utilizó para calcular la energía neta del sector residencial en cada AH (MJ/año), la cual surge de multiplicar la energía específica (MJ/año*viv.) por dicha variable (viviendas). Para ello, primero se utilizó la información de los últimos censos disponibles, en este caso desde 1960 hasta 2010, y se construyó una curva de regresión para proyectar la información hasta 2040. Posteriormente, dado que el anclaje del estudio del sector residencial se realiza en las AH, se necesita proyectar la cantidad de viviendas para cada una de es- 
tas utilizando información georreferenciada de censos previos. Calculando la incidencia de cada $\mathrm{AH}$ en el crecimiento total de las viviendas de la ciudad durante un período intercensal y conociendo la cantidad de viviendas que se incorporarán en la ciudad entre 2015-2040, se opta por distribuir las nuevas viviendas manteniendo la incidencia histórica de cada AH en el crecimiento total.

Las proyecciones aquí obtenidas serán comunes para los escenarios "tendencial” y "eficiente".

\subsection{Metodología para la construcción del escenario "tendencial"}

El escenario "tendencial" referencia la evolución probable de la demanda y de la oferta energética para los próximos años en el caso de estudio. Este se inscribe en la categoría de los escenarios exploratorios, dado que describen, a partir de una situación presente y de tendencias dominantes, una serie de hechos que conducen de forma lógica a un futuro verosímil y probable (Godet, 2000).

Al igual que en la construcción del "año base”, el abordaje para la construcción del escenario "tendencial" (ET) utiliza los niveles de análisis descriptos en la metodología general, donde se requiere un descenso de escalas que permita abarcar las distintas variables e indicadores, para luego integrar la información en las escalas superiores. En este caso, únicamente se desciende desde el nivel global hasta el sectorial, para luego realizar la integración.

\section{ET. Nivel de análisis global}

En el nivel de análisis global a escala urbana $\left(\mathrm{NAG}_{\mathrm{URB}}\right)$, se deben elaborar proyecciones del consumo de energía específica (MJ/año*hab.) para los sectores de consumo CISGyO, alumbrado público y transporte. Para ello, se utiliza un modelo de regresión lineal múltiple que considera las variables sintetizadas en la tabla 2 (modelo 'Top-Down'). Por su parte, se elabora la proyección tendencial de la oferta energética, en la cual se establecen las trayectorias probables de las fuentes primarias/secundarias y de los centros de transformación en el área de estudio. 


\section{Tabla 2}

Variables utilizadas para elaborar proyecciones de demanda de energía de los sectores no residenciales en el escenario "tendencial"

\begin{tabular}{l|ll}
\hline & \multicolumn{2}{c}{ Variables } \\
\hline Dependiente & Energía/hab. & Unidades de energía por habitante \\
\hline Independiente & PBI & $\begin{array}{l}\text { Producto bruto interno nacional } \\
\text { a precios corrientes de 2014. }\end{array}$ \\
\hline & Cr02 & $\begin{array}{l}\text { Variable dummy para el año 2002 } \\
\text { que presenta una fuerte caída en el PBI } \\
\text { y en la demanda energética ocasionada } \\
\text { por la crisis económica, política y social } \\
\text { de diciembre de 2001. }\end{array}$ \\
\hline & Variable dummy entre los años 2003 y \\
& 2015 para representar el crecimiento \\
& & económico en la postconvertibilidad. \\
\hline & Variable dummy en el año 2009 para modelar \\
& el impacto de la crisis internacional de 2008, \\
& reflejada en una caída del PBI. \\
\hline
\end{tabular}

En el nivel de análisis global del sector residencial $\left(\mathrm{NAG}_{\mathrm{RES}}\right)$, se utiliza una técnica híbrida que combina las aproximaciones 'Top-Down' y 'Bottom-Up' para obtener proyecciones de energía específica desagregadas por fuentes y por usos (MJ/año*viv.) (figura 7). La aproximación ‘Top-Down' es similar al enunciado en la tabla 3, a diferencia de que como variable dependiente se utiliza el indicador de energía por vivienda. La aproximación 'Bottom-Up' permite plantear hipótesis sobre la futura penetración y consumo de los diferentes equipos que totalizarán la demanda domiciliaria en 2040. A partir del ajuste de los valores desagregados de penetración y consumo, basados en encuestas previas y en la evolución del nivel de ventas de artefactos, se debe alcanzar una sumatoria que sea concordante con la aproximación econométrica, para luego descender al nivel de análisis sectorial. 


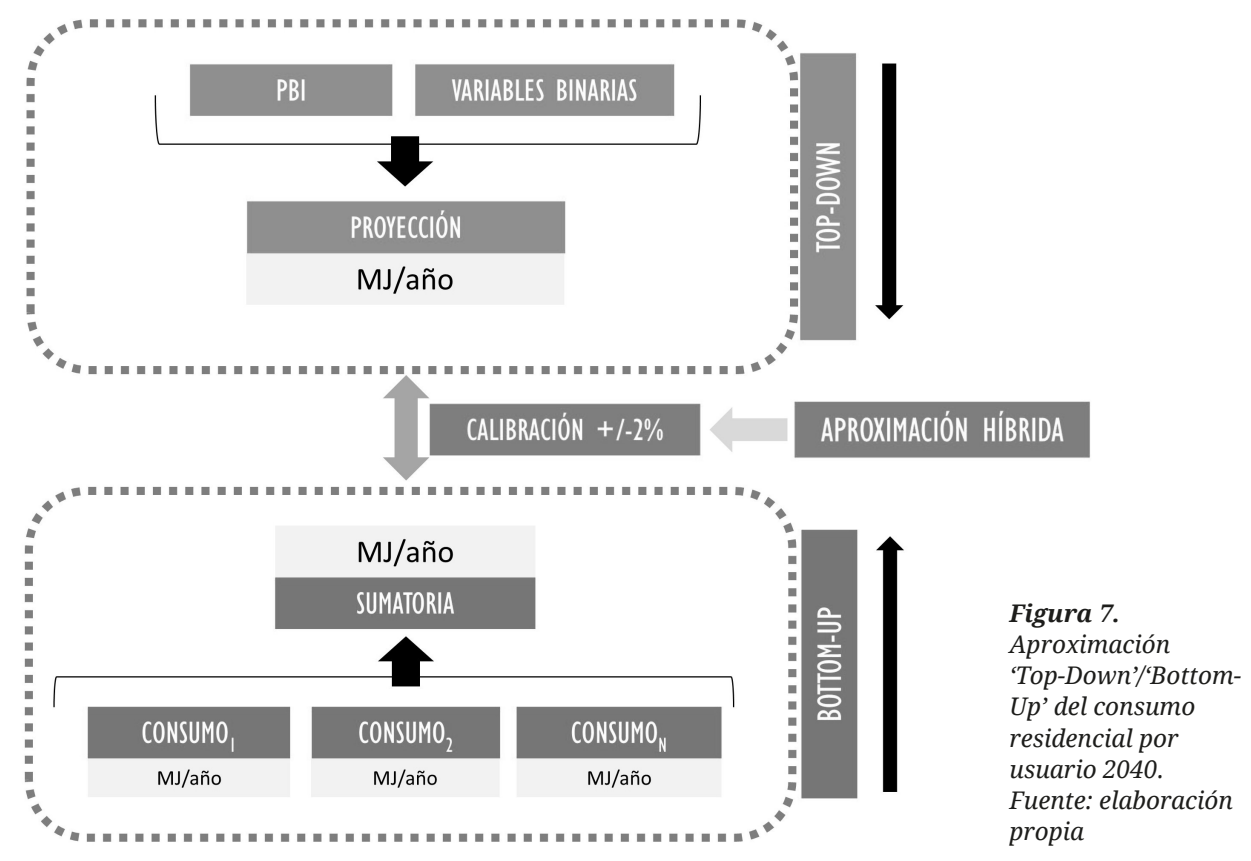

\section{ET. Nivel de análisis sectorial}

Con los valores de incremento/decrecimiento de penetración y cantidad de equipos estimados para el año 2040 en el nivel $\left(\mathrm{NAG}_{\mathrm{RES}}\right.$ ), se desciende al nivel de análisis sectorial constituido por las AH. En cada una de estas, se aplican los valores de incremento/decrecimiento sobre aquellos valores obtenidos en el "año base" y, de esta forma, se estima el consumo desagregado por fuentes y por usos para el año 2040 (figura 6). En última instancia, con la proyección de cantidad de viviendas por AH es posible calcular la demanda neta anual de cada una de las áreas. 
ET. Integración de la información: en la instancia de integración de la información se alcanza nuevamente el nivel de análisis global. En $\left(\mathrm{NAG}_{\mathrm{RES}}\right)$ se integran todas las áreas homogéneas (AH) para conformar la totalidad del sector residencial. En $\left(\mathrm{NAG}_{\mathrm{URB}}\right)$ se integran todos los sectores de la demanda y la oferta energética, cuyos valores son introducidos en el software LEAP para la elaboración del escenario "tendencial", utilizando la estructura de datos ingresada en el "año base".

\subsection{Metodología para la construcción del escenario "eficiente"}

El escenario "eficiente" ensaya medidas de mejoramiento energético y se incluye dentro de la categoría de escenarios de anticipación o normativos, los cuales se construyen a partir de imágenes alternativas de futuros deseables (GoDET, 2000). Estos se conciben de modo retrospectivo, construyendo objetivos a futuro y analizando sus trayectorias hasta el presente. Este escenario tiene como punto de partida el "año base", del cual hereda su estructura de datos, y el escenario "tendencial", del cual hereda los valores de ciertas variables que se determinan fijas. En esta instancia es posible instrumentar y evaluar propuestas tendientes a mejorar la habitabilidad del sector residencial, optimizar los patrones de consumo y disminuir la dependencia de los combustibles fósiles.

El abordaje para construir el escenario "eficiente" (EF) también adopta parte de los diferentes niveles de análisis descriptos previamente: comienza en los niveles globales, desciende hasta el nivel sectorial, para luego integrar la información.

\section{EF. Nivel de análisis global}

En cuanto al nivel global a escala urbana, $\left(\mathrm{NAG}_{\mathrm{URB}}\right.$ ), se incluyen hipótesis globales de ahorros porcentuales respecto de la demanda tendencial de energía específica (MJ/año*hab.) para los sectores no residenciales (CISGyO, alumbrado público y transporte). En el presente trabajo, estos sectores se abordan sin desagregación; sin embargo, es posible profundizar en su análisis tanto como el estudio lo requiera y ensayar medidas específicas para cada uno de estos. Para obtener la demanda neta anual de cada sector, se utiliza la serie de población del área de estudio. De esta manera, se construye un escenario integral, donde se simulan estrategias de uso eficiente de la energía en todos los sectores de consumo. 
Por su parte, se introducen las hipótesis de cambio que se desean evaluar respecto de la oferta energética y se determinan las trayectorias deseables de la oferta primaria/secundaria y de los centros de transformación en el área de estudio.

En el caso del análisis global del sector residencial $\left(\mathrm{NAG}_{\mathrm{RES}}\right)$, se requiere un relevamiento exhaustivo de las posibles medidas que implementar en materia de mejoramiento de envolvente edilicia , sustitución de equipamiento e incorporación de energías renovables, haciendo especial énfasis en determinar los valores de ahorro de energía para el caso de estudio. Para ello, se analizan planes, políticas y medidas aplicadas a nivel nacional e internacional factibles de introducir en el caso de estudio considerando mecanismos de implementación, viabilidad técnica, reducciones de energía y emisiones de $\mathrm{CO}_{2}$, costos, entre otros aspectos. Dicho relevamiento fue realizado en el trabajo final de la Especialización en Energías Renovables de la UNSa (CHÉvEz, 2016).

\section{EF. Nivel de análisis sectorial}

Una vez alcanzado el nivel sectorial, se propone para cada AH la aplicación de medidas de mejoramiento energético específicas basadas en el diagnóstico elaborado en el "año base", en el cual se detectaron los aspectos críticos de la demanda energética, como así también las oportunidades y dificultades que presenta cada una de ellas. Para la incorporación de los valores que representa cada una de las medidas se utiliza el programa de cálculo CC-Área (figura 6). Este permite insertar estrategias de mejoramiento de la envolvente edilicia, recambios en el equipamiento domiciliario e instalaciones de energías renovables.

\section{EF. Integración de la información}

En $\left(\mathrm{NAG}_{\mathrm{RES}}\right)$ se integran todas las áreas homogéneas $(\mathrm{AH})$. En ( $\left.\mathrm{NAG}_{\mathrm{URB}}\right)$ se integran todos los sectores de la demanda y la oferta energética, cuyos valores son introducidos en LEAP para la elaboración del escenario "eficiente". Para ello se utiliza la estructura de datos ingresada en el "año base” y, a su vez, se mantienen fijas algunas variables introducidas en el escenario "tendencial”.

\section{Análisis de resultados y comparativa entre escenarios}

El presente apartado incorpora una sección metodológica, en la cual se describen los métodos para la visualización de resultados y la comparación de escenarios. Posteriormente, 
se presenta una sección con salidas gráficas obtenidas de la implementación del modelo en el caso concreto de aplicación (ciudad de La Plata).

\subsection{Visualización de resultados y método para la comparativa de escenarios}

A partir de la carga de datos de los escenarios en LEAP, es posible visualizar la sección 'Resultados', que es una herramienta que permite analizar la información, individual o comparada, representada en forma de gráficos o tablas. Para la visualización de resultados es posible organizar la información por fuentes energéticas, ramas, escenario y por año, bajo sus diferentes combinaciones. Por ende, allí se pueden analizar los resultados de la simulación de los distintos escenarios. Por ejemplo, en la rama de 'Demanda' se puede obtener el nivel

\section{Tabla 3}

Indicadores para realizar la comparativa entre escenarios

\begin{tabular}{|c|c|c|c|c|}
\hline & Indicador & Definición & Unidad & Gráfico radial \\
\hline 1 & $\begin{array}{l}\text { Nivel de fosi- } \\
\text { lización de la } \\
\text { matriz eléctrica }\end{array}$ & $\begin{array}{l}\text { \% de electricidad genera- } \\
\text { da a partir de combusti- } \\
\text { bles fósiles en el año } 2040 \text {. }\end{array}$ & $\%$ & \\
\hline 2 & $\begin{array}{l}\text { Emisiones } \\
\text { de GEI }\end{array}$ & $\begin{array}{l}\text { Sumatoria de emisiones } \\
\text { (CO2 equivalente) durante } \\
2014-2040 .\end{array}$ & $\begin{array}{c}\text { t CO2 } \\
\text { eq. }\end{array}$ & \\
\hline 3 & $\begin{array}{c}\text { Costo } \\
\text { acumulado por } \\
\text { MWh generado }\end{array}$ & $\begin{array}{l}\text { Cociente entre el costo y la } \\
\text { cantidad de energía eléc- } \\
\text { trica generada entre } 2014 \text { - } \\
2040 .\end{array}$ & $\begin{array}{l}\text { USD/ } \\
\text { MWh }\end{array}$ & \\
\hline 4 & $\begin{array}{l}\text { Demanda de } \\
\text { energía neta }\end{array}$ & $\begin{array}{l}\text { Sumatoria de todas las } \\
\text { fuentes utilizadas por la } \\
\text { demanda entre 2014-2040. }\end{array}$ & GJ & $\cdots$ \\
\hline 5 & $\begin{array}{l}\text { Requerimientos } \\
\text { de combustibles } \\
\text { fósiles (energía } \\
\text { primaria) }\end{array}$ & $\begin{array}{l}\text { Sumatoria de petróleo, } \\
\text { carbón y gas, utilizados en } \\
\text { la rama de 'Recursos’ en- } \\
\text { tre 2014-2040. }\end{array}$ & GJ & $\begin{array}{l}\text { Escenario tendencial } \\
\text { Escenario eficiente }\end{array}$ \\
\hline
\end{tabular}




\section{Desarrollo metodológico para la construcción de escenarios urbano-energéticos de largo plazo}

de desagregación que se requiera, analizar sectores particulares, la participación de combustibles, como así también establecer comparativas entre escenarios, entre otras opciones.

En última instancia, para lograr una comparativa ágil entre escenarios, se propone un método a partir de cinco indicadores normalizados (0-1) que permiten caracterizar y comprender el impacto de cada escenario a partir de un gráfico radial (tabla 3). Este método comparativo permite evaluar el impacto de las diferentes medidas, lo que habilita a reformularlas y volver a ensayarlas en los escenarios para usar más eficientemente los recursos.

\subsection{Ejemplo de aplicación: resultados obtenidos en un caso de estudio concreto}

Tal como se mencionó, la presente metodología fue implementada en un caso de estudio real (ciudad de La Plata), en el marco de un trabajo de tesis doctoral (CHÉvez, 2017). A continuación, se exponen algunos de los resultados obtenidos en dicho trabajo, a los efectos de ejemplificar las salidas y los análisis posibles de realizar.

En la figura 8 se observa la demanda neta de energía hasta 2040 desagregada por fuentes para la ciudad de La Plata, y se identifica que para el último año de la serie, el escenario "eficiente", que incluyó numerosas medidas de mejoramiento, presenta un consumo un $18,84 \%$ menor respecto del "tendencial”.

Demanda "tendencial" por fuentes

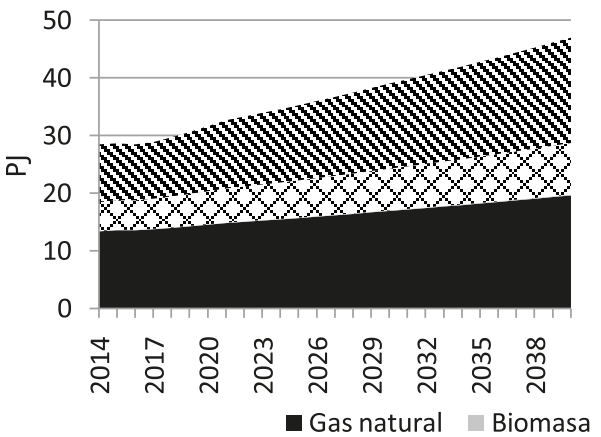

Demanda "eficiente" por fuentes

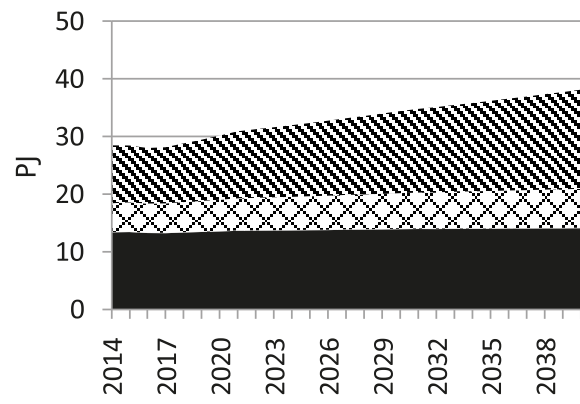

$\times$ Electricidad $\mathbf{s}$ Comb. líquidos
Figura 8. Demanda neta discriminada por fuentes: Escenario "tendencial" (izq.) y Escenario "eficiente" (der.). Fuente: elaboración propia 
Por otro lado, la figura 9 muestra los resultados de la proyección de la demanda eléctrica en ambos escenarios hasta el año 2040, dividida por sectores de consumo. Se observa que en 2040 el escenario "eficiente" consume un 24,6 \% menos que el "tendencial", con valores netos de 1874 GWh y 2486 GWh, respectivamente.

Figura 9.

Evolución de la demanda neta eléctrica para la ciudad de La Plata (1996-2040).

Fuente: elaboración propia

\section{Demanda eléctrica neta ET 1996-2040}

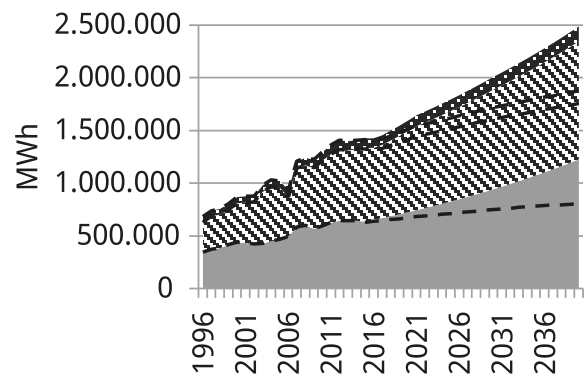

Residencial

Alumbrado

\section{Demanda eléctrica neta EF 1996-2040}

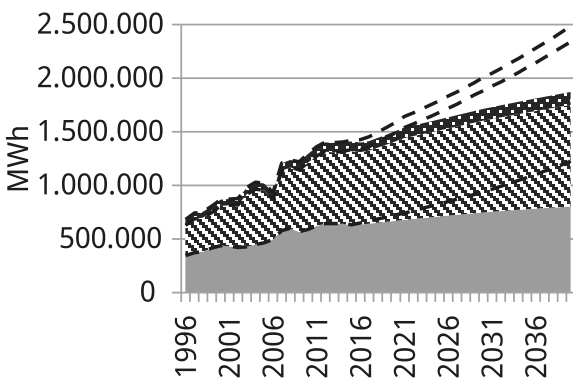

Residencial мแN CISGyO - - - "Tendencial"

La demanda eléctrica de cada escenario es abastecida por matrices de generación diferentes, ya sea porque los ingresos de potencia son menores en el escenario "eficiente" respecto del "tendencial”, o porque en el "eficiente” se planteó la integración de energías renovables. En consecuencia, es posible obtener el costo total de la generación eléctrica para los distintos escenarios, considerando los costos de capital (inversiones), combustibles y operación y mantenimiento (fijos y móviles). A partir de este análisis se obtuvo que, para el caso de estudio, el costo acumulado (2014-2040) para la generación eléctrica "tendencial” sería de 3.382 millones de dólares y el "eficiente" sería de 2.635 millones de dólares (figura 10). Por su parte, si se calcula el costo de generación promedio para todo el período simulado, en el escenario "tendencial" se obtiene un valor de 59,42 USD/MWh y en el escenario "eficiente", un valor de 53,46 USD/MWh. 


\section{Desarrollo metodológico para la construcción de escenarios urbano-energéticos de largo plazo}

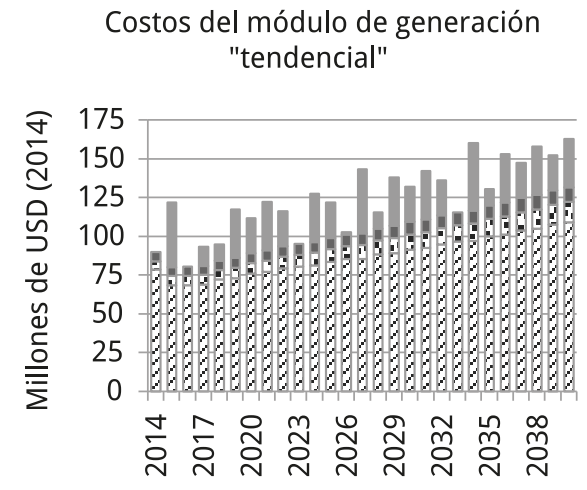

n Costos variables de O\&M Costos de capital

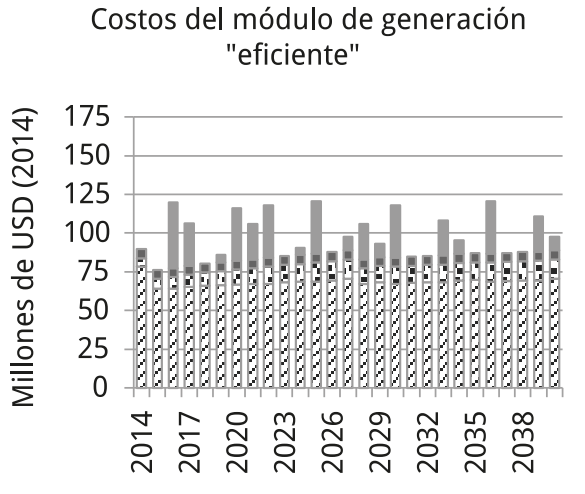

$\square$ Costos de combustibles Costos fijos de O\&M

En relación con el consumo energético del sector residencial (figura 11), en este caso, considerando la sumatoria de todos los vectores, el escenario "eficiente" presenta, en el año 2040, una demanda neta un 36,40 \% menor respecto del escenario "tendencial" (10,77 PJ contra 16,95 PJ). En dicha figura también es posible identificar el peso de las distintas AH en la obtención del mencionado ahorro energético.

Demanda neta (ET) residencial

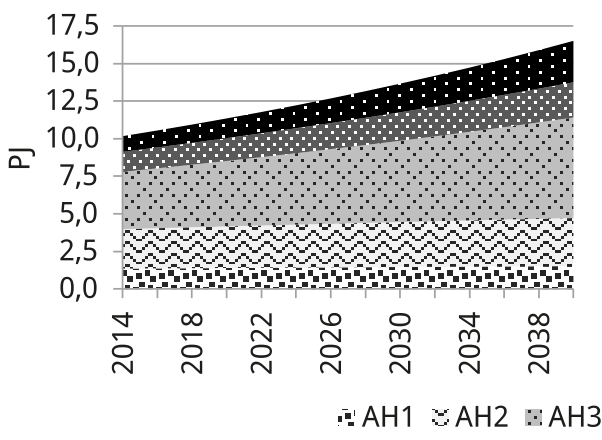

\section{Demanda neta (EF) residencial}

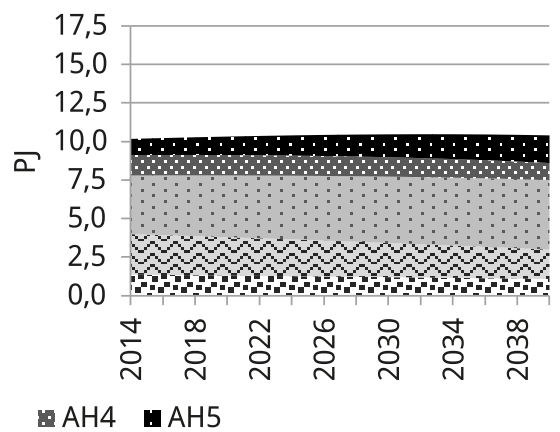

Figura 10. Costos de generación para la ciudad de La Plata en ambos escenarios. Fuente: elaboración propia
Figura 11. Evolución de la demanda neta de para las cinco $\mathrm{AH}$ en ambos escenarios. Fuente: elaboración propia 
Los resultados hasta aquí expuestos son ilustrativos de las posibilidades que ofrece la metodología para analizar el impacto de los escenarios sobre diversas variables. Tal como se verificó, es posible cuantificar la demanda de energía desagregada por fuentes y por sectores de consumo, analizar los costos del módulo de generación eléctrica, profundizar en la demanda del sector residencial, entre muchas otras opciones que se excluyen del presente trabajo por motivos de extensión y que se pueden consultar en el trabajo de referencia (CHÉvez, 2017).

\section{Conclusiones}

La aplicación de la metodología y el esquema de abordaje "en cascada", ante un objeto de estudio complejo como lo es la ciudad, lograron responder satisfactoriamente a los objetivos planteados en el inicio del trabajo. Estos proponían elaborar una exposición clara y consistente de los aspectos metodológicos a los efectos de posibilitar la evaluación y validación los distintos métodos, técnicas y datos utilizados, dejando la metodología "abierta” y susceptible de ser mejorada, ampliada, ajustada con nuevos conjuntos de datos, para volver más precisos los resultados y/o para que sea adoptada por otros casos de estudio.

A partir de la implementación de la presente metodología, es posible examinar la interacción entre variables económicas, socio-demográficas, morfológicas-urbanas, de equipamiento y de demanda y oferta energética, en diferentes escalas territoriales y horizontes temporales. Esto permite la construcción de una plataforma técnico-instrumental que, en última instancia, posibilita el planteo de escenarios urbano-energéticos. A partir de ellos es viable establecer escenarios futuros alternativos, tanto posibles como deseables, cuya formulación se basa en hipótesis, observaciones y diagnósticos pormenorizados del objeto de estudio, y a partir de los cuales es posible obtener elementos de vital importancia para establecer lineamientos y orientar políticas energéticas.

Asimismo, tal como se destaca en la revisión de antecedentes, existe una extensa bibliografía que desarrolla estudios con base en la técnica de escenarios. Allí se observaron ciertas diferencias en el enfoque y en el alcance de los trabajos; sin embargo, se verificó que es poco frecuente detectar abordajes integrales que incluyan múltiples medidas de mejoramiento, tanto en la oferta como en la demanda, y que consideren las diferencias que presentan los distintos sectores que conforman la ciudad. En ese sentido, el presente trabajo pretendió 
abordar dicha vacancia, y entendemos que este objetivo se alcanzó de manera satisfactoria, puesto que la metodología permite el ensayo de medidas de mejoramiento de la envolvente edilicia, del equipamiento domiciliario y la incorporación de energías renovables, siendo posible cuantificar ahorros potenciales en los distintos sectores urbanos. En este sentido, la sección 5.2 incluyó resultados de la implementación de la presente metodología en un caso concreto de aplicación — la ciudad de La Plata—, donde se verificó la versatilidad del modelo para analizar el impacto de cada uno de los escenarios sobre distintas variables.

Asimismo, la utilización de diferentes escalas de abordaje permitió organizar y poner en discusión los métodos, técnicas y datos utilizados. Esto genera la posibilidad de extrapolar esta metodología a distintos casos de estudio, con sus adecuaciones pertinentes, por lo que se trata de un desarrollo que contribuye a la tendencia incipiente en la que las ciudades están comenzando a asumir el rol de impulsar diferentes medidas de mejoramiento energético.

\section{Bibliografía}

ABDURAFIKOV, Rinat; GRAHN, Elina; KANNARI, Lotta; YPYÄ, Joel; KAUKONEN, Sampo; HEIMONEN, Ismoy y PAIHO, Satu (2017). "An analysis of heating energy scenarios of a Finnish case district”. Sustainable Cities and Society (32), pp. 56-66.

AMADO, Miguel; POGGI, Francesca y AMADO, Antonio (2016). "Energy efficient city: a model for urban planning”. Sustainable Cities and Society (26), pp. 476-485.

BARRERA, Mariano; SABBATELLA, Ignacio y SERRANI, Esteban (2012). Historia de una privatización. Cómo y por qué se perdió YPF. Buenos Aires, Capital Intelectual.

BRITO, M.; FREITAS, S.; GUIMARÃES, S., CATITA, C. y REDWEIK, P. (2017). "The importance of facades for the solar PV potential of a Mediterranean city using LiDAR data”. Renewable Energy (111), pp. 85-94.

DISCOLI, Carlos (2009). Metodología para el diagnóstico urbano-energético-ambiental en aglomeraciones intermedias. El caso del Gran La Plata. La Plata, EDULP.

CHÉVEZ, Pedro (2016). "Análisis de medidas de eficiencia energética y energías renovables en el sector residencial”. [Tesis de Especialización en Energías Renovables]. UNSa, Facultad de Ciencias Exactas.

CHÉVEZ, Pedro (2017). "Construcción de escenarios urbano-energéticos a partir de la implementación de estrategias de eficiencia energética y energías renovables en el sector residencial”. [Tesis de Doctorado en Ciencias]. UNSa, Facultad de Ciencias Exactas. 
CORTEKAR, Jörg.; BENDER, Steffen y GROTH, Markus (2016). "Why climate change adaptation in cities needs customized and flexible climate services”. Climate Services (4), pp. 42-51.

DECRETO 134 (2015). “Emergencia energética. Boletín Oficial”. Buenos Aires, Argentina, 16 de diciembre, 2015.

DPE (2016). Proyecciones de población por Municipio. Provincia de Buenos Aires 2010-2025. La Plata, Ministerio de Economía de la Provincia de Buenos Aires.

DROUILLES, Judith; LUFKIN, Sophiey REY, Emmanuel (2017). "Energy transition potential in peri-urban dwellings: Assessment of theoretical scenarios in the Swiss context”. Energy and Buildings (148), pp. 379-390.

EICKER, Ursula; NOUVEL, Romain; DUMINIL, Eric y COORS, Volker (2014). "Assessing passive and active solar energy resources in cities using 3D city models”. Energy Procedia (57), pp. 896-905.

FMI (2016). "World Economic Outlook Database. World Economic and Financial Surveys”. Washington D.C., Fondo Monetario Internacional.

GHIASSI, Neday y MAHDAVI, Ardeshir (2017). "Reductive bottom-up urban energy computing supported by multivariate cluster analysis”. Energy and Buildings (144), pp. 372-386.

GIRARDIN, Luc; MARECHAL, François; DUBUIS, Matthias; CALAME-DARBELLAY, Nicoley FAVRAT, Daniel (2010). "EnerGis: A geographical information based system for the evaluation of integrated energy conversion systems in urban areas”. Energy (35), pp. 830-840.

GODET, Michel (2000). La Caja de Herramientas de la Prospectiva Estratégica. París, Laboratoire d'Investigation Prospective et Stratégique.

GUZOWSKI, Carina (2015). "La gestión de los mercados de generación eléctrica en la región sudamericana: la especificidad del caso argentino”. [Tesis de Doctorado en Economía]. UNS, Departamento de Economía.

HOWARD, B.; PARSHALL, L.; THOMPSON, J.; HAMMERB, S. y MODIA, V. (2012). "Spatial distribution of urban building energy consumption by end use”. Energy and Buildings (45), pp.141-151. 
INDEC (2016). “Censo Nacional de Población, Hogares y Viviendas 2010. Censo del Bicentenario. Base de datos REDATAM". CABA, Instituto Nacional de Estadísticas y Censos.

MINEM (2016). "Balance Energético Nacional 2014”. Buenos Aires, Ministerio de Energía y Minería.

MÖRTBERG, Ulla; GOLDENBERG, Romain; KALANTARI, Zahra; KORDAS, Olga; DEAL, Brian; BALFORS, Berity CVETKOVIC, Vladimir (2017). "Integrating ecosystem services in the assessment of urban energy trajectories. A study of the Stockholm Region”. Energy Policy (100), pp. 338-349.

OCDE (2012). Economic Outlook, Volume 2012. Issue 1. París, Organisation for Economic Cooperation and Development.

PÉREZ LÓPEZ, César (2005). Muestreo estadístico. Conceptos y problemas resueltos. Madrid, Pearson.

RODRÍGUEZ, Lucas; MARTINI, Irene y DISCOLI, Carlos (2016). "Energy storage for residential dwellings. Methodology to improve energy efficiency and habitability". Journal of Energy Storage (8), pp. 99-110.

RUGGERI, Emilia N. (2017). "El sistema eléctrico argentino: construcción, crisis y reconfiguración”. En: Actas de la VII Jornada de Becarios y Tesistas UNQ. Quilmes, UNQ.

SVEINBJÖRNSSON, Dadi; AMER-ALLAM, Sara; BAVNHøJ, Anders; ALGREN, Louiy SCHRøDER PEDERSEN, Allan (2017). "Energy supply modelling of a low-CO2 emitting energy system: Case study of a Danish municipality”. Applied Energy (195), pp. 922-941.

VIEGAS, Graciela (2010). "Evaluación del potencialenergético e intervenciones de mejoramiento del entorno edilicio en áreas urbanas de media y baja consolidación”. [Tesis de Doctorado en Ciencias]. UNSa, Facultad de Ciencias Exactas.

VIEGAS, Graciela; CHÉVEZ, Pedro; DISCOLI, Carlos y SAN JUAN, Gustavo (2017). “Comportamiento energético de mosaicos urbanos representativos de la ciudad de La Plata (Bs. As., Argentina) en función de las condiciones térmicas externas”. En Actas del X ELACAC. Porto Alegre, ANTAC.

WORLD ENERGY COUNCIL (2016). Perspective input into the World Energy Council Scenarios: "Innovating Urban Energy". Londres, World Energy Council. 
Artículos Arbitrados 